\title{
Vitamin E sequestration by liver fat in humans
}

Pierre-Christian Violet, ${ }^{1}$ Ifechukwude C. Ebenuwa, ${ }^{1}$ Yu Wang, ${ }^{1}$ Mahtab Niyyati, ${ }^{1}$ Sebastian J. Padayatty, ${ }^{1}$ Brian Head, ${ }^{2}$ Kenneth Wilkins, ${ }^{3}$ Stacey Chung, ${ }^{4}$ Varsha Thakur, ${ }^{4}$ Lynn Ulatowski, Jeffrey Atkinson, ${ }^{5}$ Mikel Chelfi, ${ }^{5}$ Sheila Smith, ${ }^{1}$ Hongbin Tu, ${ }^{1}$ Gerd Bobe, ${ }^{2}$ Chia-Ying Liu, ${ }^{6}$ David W. Herion, ${ }^{7}$ Robert D. Shamburek, ${ }^{8}$ Danny Manor, ${ }^{4}$ Maret C. Traber, ${ }^{2}$ and Mark Levine ${ }^{1}$

'Molecular and Clinical Nutrition Section, Intramural Research Program, National Institute of Diabetes and Digestive and Kidney Diseases, NIH, Bethesda, Maryland, USA. 'Linus Pauling Institute, Oregon State University, Corvallis, Oregon, USA. ${ }^{3}$ Office of the Director, National Institute of Diabetes and Digestive and Kidney Diseases, NIH, Bethesda, Maryland, USA. ${ }^{4}$ Department of Pharmacology and Department of Nutrition, School of Medicine, Case Western Reserve University and the Case Comprehensive Cancer Center, Cleveland, Ohio, USA. ${ }^{5}$ Department of Chemistry, Brock University, Saint Catharines, Ontario, Canada. ${ }^{6}$ Radiology and Imaging Sciences and ${ }^{7}$ Clinical Research Informatics, Clinical Center, $\mathrm{NIH}$, Bethesda, Maryland, USA. ${ }^{8}$ Cardiovascular Branch, Intramural Research Program, National Heart, Lung, and Blood Institute, $\mathrm{NIH}$, Bethesda, Maryland, USA.

BACKGROUND. We hypothesized that obesity-associated hepatosteatosis is a pathophysiological chemical depot for fat-soluble vitamins and altered normal physiology. Using $\alpha$-tocopherol (vitamin E) as a model vitamin, pharmacokinetics and kinetics principles were used to determine whether excess liver fat sequestered $\alpha$-tocopherol in women with obesity-associated hepatosteatosis versus healthy controls.

METHODS. Custom-synthesized deuterated $\alpha$-tocopherols $\left(d_{3}-\right.$ and $d_{6}-\alpha$-tocopherols) were administered to hospitalized healthy women and women with hepatosteatosis under investigational new drug guidelines. Fluorescently labeled $\alpha$-tocopherol was custom-synthesized for cell studies.

RESULTS. In healthy subjects, $85 \%$ of intravenous $d_{6}-\alpha$-tocopherol disappeared from the circulation within 20 minutes but reappeared within minutes and peaked at 3-4 hours; $d_{3}$ - and $d_{6}-\alpha$-tocopherols localized to lipoproteins. Lipoprotein redistribution occurred only in vivo within 1 hour, indicating a key role of the liver in uptake and re-release. Compared with healthy subjects who received $2 \mathrm{mg}$, subjects with hepatosteatosis had similar $d_{6}$ - $\alpha$-tocopherol entry rates into liver but reduced initial release rates $(P<0.001)$. Similarly, pharmacokinetics parameters were reduced in hepatosteatosis subjects, indicating reduced hepatic $d_{6}$ - $\alpha$-tocopherol output. Reductions in kinetics and pharmacokinetics parameters in hepatosteatosis subjects who received $2 \mathrm{mg}$ were echoed by similar reductions in healthy subjects when comparing 5- and 2-mg doses. In vitro, fluorescentlabeled $\alpha$-tocopherol localized to lipid in fat-loaded hepatocytes, indicating sequestration.

CONCLUSIONS. The unique role of the liver in vitamin E physiology is dysregulated by excess liver fat. Obesity-associated hepatosteatosis may produce unrecognized hepatic vitamin $\mathrm{E}$ sequestration, which might subsequently drive liver disease. Our findings raise the possibility that hepatosteatosis may similarly alter hepatic physiology of other fat-soluble vitamins.

TRIAL REGISTRATION. ClinicalTrials.gov, NCT00862433.

FUNDING. National Institute of Diabetes and Digestive and Kidney Diseases and NIH grants DK053213-13, DK067494, and DK081761.

for Clinical Investigation.

Submitted: September 10, 2019

Accepted: November 26, 2019

Published: December 10, 2019 .

Reference information: /CI Insight. 2020;5(1):e133309.

https://doi.org/10.1172/jici.

insight.133309.

\section{Introduction}

The global obesity epidemic has sobering consequences to upend human health (1-3). Especially concerning is obesity-associated hepatosteatosis (HS), or nonalcoholic fatty liver. Nonalcoholic fatty liver is already the most common precursor of chronic liver disease in the Americas and Western Europe (4-6). 
Specifically, nonalcoholic fatty liver precedes nonalcoholic steatohepatitis (NASH) and ultimately cirrhosis and hepatocellular carcinoma. The penetrance of this spectrum of liver diseases is increasing rapidly and concurrently with obesity and diabetes, with staggering consequences for morbidity, mortality, and health care costs. For NASH treatment, some patients had reduced inflammation and hepatocyte injury with pharmacological doses of vitamin E ( $\alpha$-tocopherol), but fibrosis leading to cirrhosis was not improved (7, $8)$. For NASH prevention, the only current strategy is maintenance of ideal body weight $(9,10)$. Nevertheless, prevention strategies are gold standards to avert the need for treatment, disease consequences, and huge societal cost.

Although indirect, there are several clues that $\alpha$-tocopherol, distinct from its role in partially treating disease, could prevent NASH. First, the liver participates in $\alpha$-tocopherol physiology. Dietary $\alpha$-tocopherol is trafficked out of the liver on lipoproteins, with involvement of the hepatocyte-localized $\alpha$-tocopherol transfer protein (TTP) $(11,12)$. Second, because $\alpha$-tocopherol is fat soluble, excess fat in hepatocytes could serve as an unintended chemical sink. By sequestering $\alpha$-tocopherol so that it is biologically less available or unavailable, liver fat could cause a local deficiency of the vitamin within the liver, over years, leading to progression of liver disease: from nonalcoholic fatty liver, to nonalcoholic fatty liver disease, and finally to $\mathrm{NASH} /$ hepatocellular carcinoma. Third, use of $\alpha$-tocopherol in treatment of NASH $(13,14)$ hints at an underlying local nutritional deficiency of $\alpha$-tocopherol in the liver.

With this background, we hypothesized that liver fat accompanying obesity could act as an unintended chemical depot for the lipophilic $\alpha$-tocopherol. If correct, then release of $\alpha$-tocopherol from the liver could be attenuated, and its physiological efficacy reduced. Essential prerequisites for testing these concepts are development and use of new approaches to characterize $\alpha$-tocopherol physiology in both healthy people and in people with obesity-associated hepatosteatosis, without evidence of hepatitis. We used specially synthesized deuterated $\alpha$-tocopherol preparations as investigational new drug (IND) products for simultaneous oral and intravenous administration in humans (15-18). These materials were used to perform kinetics and pharmacokinetics studies in healthy women and those with HS. Deuterated $\alpha$-tocopherol doses were selected to be similar to amounts in foods. Preclinical studies were performed in animals and in cultured hepatocytes using fluorescently labeled $\alpha$-tocopherol $(19,20)$.

\section{Results}

Plasma concentrations of $d_{6}$ and $d_{3}$ - $\alpha$-tocopherol and redistribution into lipoproteins in healthy women. To characterize vitamin $\mathrm{E}$ trafficking in healthy subjects (demographics in Table 1 and Figure 1), we used 2 custom-synthesized deuterated $\alpha$-tocopherol preparations: an oral solution of $d_{3}$ - $\alpha$-tocopherol and an intravenous small-particle (particles $<0.5 \mathrm{~nm}$ ) sterile emulsion with $\mathrm{d}_{6}$ - $\alpha$-tocopherol. Both were administered to subjects under the auspices of an approved US FDA IND application (held by ML). Deuterated tocopherols at a dose of $5 \mathrm{mg}$, an amount found in an avocado, were administered simultaneously orally and intravenously at time 0 to healthy hospitalized women (Figure 2A). Deuterated $\alpha$-tocopherols were measured in plasma for up to 72 hours (Figure $2 B$ ). Nearly $85 \%$ of intravenous $d_{6}$ - $\alpha$-tocopherol disappeared from the circulation within 20 minutes, with reappearance peaking at 3 hours and remaining steady until 8 hours (Figure $2 \mathrm{C}$ ). Reappearance of oral $\mathrm{d}_{3}$ - $\alpha$-tocopherol was slower, presumably because of the kinetics of intestinal absorption and lymphatic transport to the thoracic duct (21-23). To evaluate the trafficking of tocopherols in lipoprotein particles, we calculated percentage enrichment, which describes the percentage of deuterated $\alpha$-tocopherols compared with total $\alpha$-tocopherols on lipoproteins. Deuterated $\alpha$-tocopherols were localized to lipoproteins (VLDL, HDL, LDL), with a shift of the vitamin to HDL and LDL over the time course (Figure 2, D and E). Approximately $100 \%$ of plasma $d_{6}$ - and $d_{3}-\alpha$-tocopherols were recovered on lipoproteins, implicating liver involvement (Figure $2 \mathrm{~F}$ ). When blood samples were incubated ex vivo with $d_{6}-\alpha$-tocopherol, this shift to lipoproteins (LDL and HDL) was absent (Figure 2G). These data show that $d_{6}$ - $\alpha$-tocopherol was translocated within minutes from plasma to the liver of healthy subjects, followed by reappearance (release) in the circulation and redistribution on lipoproteins.

To evaluate whether $\alpha$-tocopherol pharmacokinetics parameters other than maximum concentration $\left(\mathrm{C}_{\max }\right)$ and AUC are influenced by administered doses of deuterated $\alpha$-tocopherol (dose effect), we performed similar studies using a lower dose $(2 \mathrm{mg}$ ) of deuterated $\alpha$-tocopherol (half an avocado) in healthy subjects. We compared $\mathrm{d}_{6}$ - $\alpha$-tocopherol pharmacokinetics parameters between 2-mg and 5-mg doses (Figure $2 \mathrm{H}$ and Supplemental Table 1; supplemental material available online with this article; https://doi. org/10.1172/jci.insight.133309DS1). As expected, results revealed reduced $\mathrm{C}_{\max }$ and $\mathrm{AUC}_{0-72 \mathrm{~h}}$ for the 2-mg 


\section{Table 1. Baseline characteristics}

\begin{tabular}{|c|c|c|c|}
\hline & Healthy 5-mg intervention & Healthy 2-mg intervention & HS 2-mg intervention \\
\hline Number of subjects & 6 & 10 & 6 \\
\hline $\mathrm{BMI} \mathrm{kg} / \mathrm{m}^{2}$ & $24(21.3-26.1)$ & $24.5(19.9-26.3)$ & $42.5(32.9-61.3)$ \\
\hline Percentage liver fat (MRS) ${ }^{A}$ & $0.5(0.3-0.9)$ & $0.6(0.3-1.1)$ & $12.1(7.2-23.9)$ \\
\hline Percentage body fat (DEXA) ${ }^{\mathrm{B}}$ & $33.9(26.6-39.4)$ & $32.3(26.6-39.4)$ & $44.3(32.9-56.5)$ \\
\hline Age (years) & $26(19-30)$ & $26(19-35)$ & $41(33-53)$ \\
\hline $\operatorname{AST}^{\mathrm{C}}(\mathrm{U} / \mathrm{L})$ & $17(12-27)$ & $17(14-27)$ & $17(12-24)$ \\
\hline FIB-4 score & $0.58(0.33-0.73)$ & $0.63(0.3-0.87)$ & $0.60(0.27-0.91)$ \\
\hline $\mathrm{TC}(\mathrm{mg} / \mathrm{dL})$ & 150 (129-181) & $150(133-181)$ & $165(142-227)$ \\
\hline $\mathrm{HDL}$ (mg/dL) & $62(49-74)$ & $56(41-81)$ & $47(34-68)$ \\
\hline LDL (mg/dL) & $73(58-124)$ & $81(58-111)$ & $94(80-145)$ \\
\hline TG (mg/dL) & $46(31-96)$ & $70(37-138)$ & $114.5(72-181)$ \\
\hline
\end{tabular}

Demographics of healthy subjects and subjects with hepatosteatosis (HS) $(n=6)$. Intervention groups included the following participants: 5 mg healthy $(n=6), 2 \mathrm{mg}$ healthy $(n=10)$, and $2 \mathrm{mg} \mathrm{HS}(n=6)$. The medians were calculated from the baseline data of the individuals participating in that group. Fibrosis-4 (FIB-4) score was calculated as follows: (age [year] x AST [U/L]) / (platelets [109/L] x ALT [U/L] 0.5 ). Values less than 1.45 have a negative predictive value of $90 \%$ to exclude advanced fibrosis (30). Data are shown as mean (range). Normal ranges: BMI (18-26.9), AST (0-32 U/L), ALT (0-33 $\mathrm{U} / \mathrm{L}$ ), platelets (173.10 $\left.-369.10^{9} / \mathrm{L}\right)$, FIB-4 score (<1.45), TC (0-150 mg/dL), HDL (40-59 mg/dL), LDL (0-129 mg/dL), and TC (0-149 mg/dL). ${ }^{A} \mathrm{Liver}$ fat was determined by magnetic resonance spectroscopy. ${ }^{B}$ Body fat was determined by dual-energy $x$-ray absorptiometry whole-body composition analyses (Hologic Discovery QDR). ${ }^{\circ}$ Three values for aspartate aminotransferase (AST) and alanine aminotransferase (ALT) were obtained 1-4 months before and during study. The overall mean for each enzyme is shown. U/L, units per liter; BMI, body mass index; TC, total cholesterol; HDL, high-density lipoprotein; LDL, low-density lipoprotein; TC, triglyceride.

dose (56\% and $60 \%$ reduction, respectively, of those during the 5 -mg dosing) in healthy subjects. All other pharmacokinetic parameters were similar (Supplemental Table 1). We also compared $d_{6}$ - $\alpha$-tocopherol initial release kinetics over the first 4 hours, when release was linear (initial rate conditions, Figure $2 \mathrm{H}$ inset) between matched healthy subjects who received $2 \mathrm{mg}$ and $5 \mathrm{mg}$ during different hospitalizations (see Methods), and found reduced release kinetics with the 2-mg dose ( $5 \mathrm{mg}, 0.195 \mu \mathrm{M} / \mathrm{h} ; 2 \mathrm{mg}, 0.083 \mu \mathrm{M} / \mathrm{h}$; 2.4-fold decrease). Taken together, these findings underscore the essential role of the liver in $\alpha$-tocopherol physiology, with similar pharmacokinetics with both 5-mg and 2-mg doses, except that the lower dose reduced initial release, $\mathrm{C}_{\max }$, and AUC.

Influence of liver fat on $d_{\sigma}-\alpha$-tocopherol plasma concentrations: pharmacokinetics and kinetics in 10 healthy and 6 $H S$ subjects. With this foundation indicating the essential role of the liver in rapidly taking up intravenously administered $\mathrm{d}_{6}-\alpha$-tocopherol and subsequently releasing it into plasma, we hypothesized that excess liver fat would sequester fat-soluble $\alpha$-tocopherol and attenuate its release from the liver to the circulation similarly to acquiring a smaller dose of vitamin $\mathrm{E}$ delivered to the liver. We tested our hypothesis by comparing pharmacokinetics and kinetics changes in healthy and HS subjects. Given similar pharmacokinetics of 2-mg and 5-mg doses (Supplemental Table 1), we chose the lower 2-mg dose because a lower dose could provide a more sensitive means to probe liver fat effects, and this is an amount found in many serving sizes of vitamin E-rich foods. Additionally, we used intravenous $\mathrm{d}_{6}-\alpha$-tocopherol to bypass confounding effects of gastrointestinal absorption with orally administered $\mathrm{d}_{3}-\alpha$-tocopherol.

Effect of liver fat on pharmacokinetics parameters of $d_{6}-\alpha$-tocopherol in healthy versus $H S$ subjects. Plasma $\mathrm{d}_{6}-\alpha$-tocopherol concentrations were measured after intravenous administration of $2 \mathrm{mg} \mathrm{d}_{6}-\alpha$-tocopherol (Figure 3A). To evaluate possible differences in $\alpha$-tocopherol liver physiology between healthy and HS subjects, we calculated and compared pharmacokinetics parameters for $\mathrm{d}_{6}-\alpha$-tocopherol in the 2 groups (Table 2). HS subjects had a $30 \%$ reduction in $\mathrm{d}_{6}-\alpha$-tocopherol $C_{\max }$ versus healthy subjects $(0.34 \pm 0.04$ $\mu \mathrm{M}$ vs. $0.48 \pm 0.02 \mu \mathrm{M}, P<0.05)$. HS subjects also had a $30 \%$ reduction in $\mathrm{d}_{6}$ - $\alpha$-tocopherol $\mathrm{AUC}_{0-8 \mathrm{~h}}$ (time to $\mathrm{C}_{\max }$ ) and $25 \%$ reduction in $\mathrm{AUC}_{0-72 \mathrm{~h}}$ (Table 2). There were no significant differences in the other $\mathrm{d}_{6}-\alpha$-tocopherol parameters $\left(\mathrm{T}_{\max }, \mathrm{K}_{\mathrm{e}}\right.$, and half-life) between HS and healthy groups (Table 2 ). Together, these findings show altered pharmacokinetics (reduced $\mathrm{C}_{\max }$ and AUC) in HS subjects in a similar pattern observed with $5 \mathrm{mg}$ versus $2 \mathrm{mg}$ (Figure $2 \mathrm{H}$ and Supplemental Table 1). 


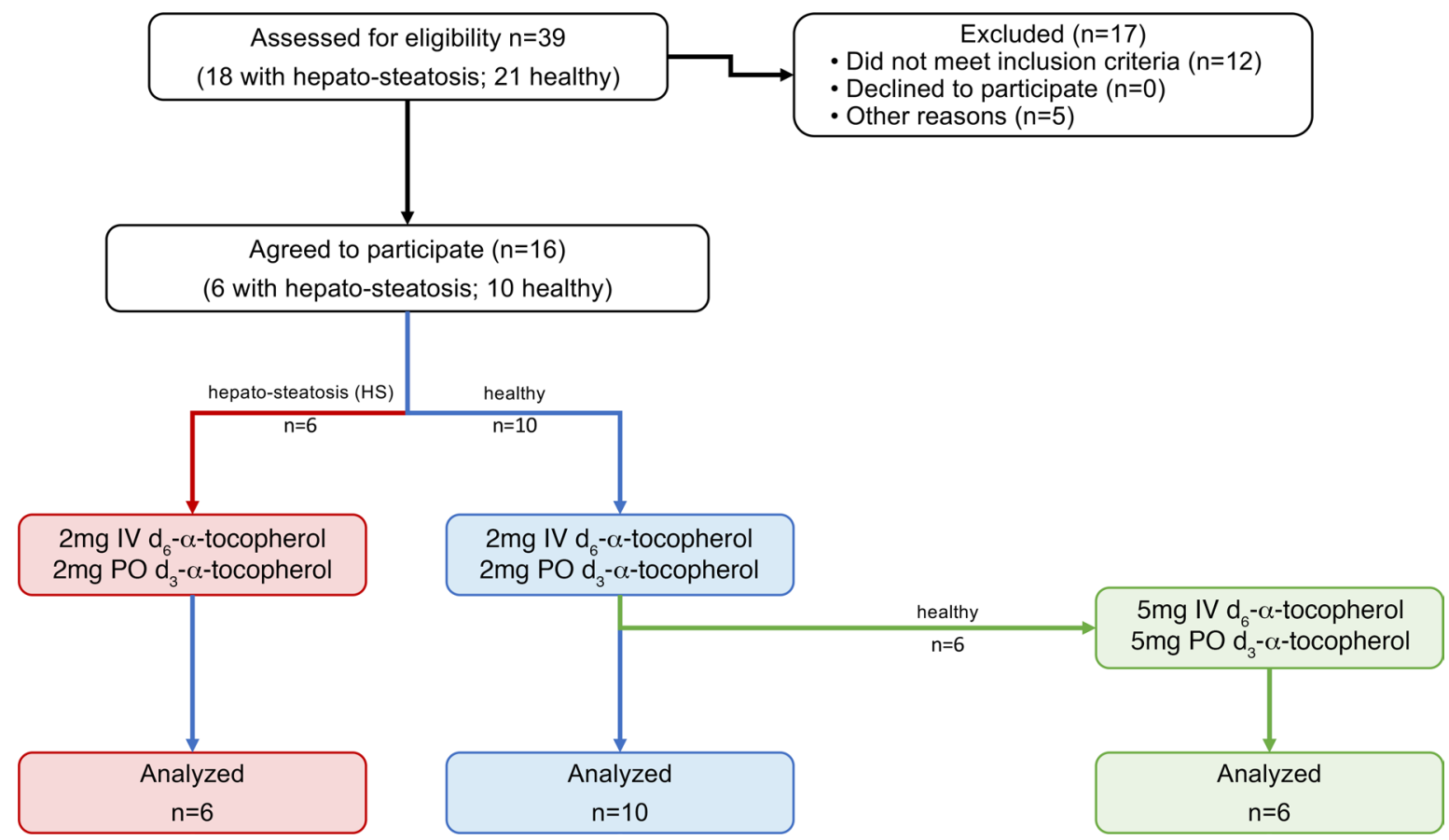

Figure 1. Recruitment scheme. The recruitment scheme is shown for this clinical intervention (NCT00862433), conducted at the NIH Clinical Research Center. Data from all participants are included in the analyses. Green indicates 6 healthy women of the 10 healthy women who received 2 $\mathrm{mg} \alpha$-tocopherol who also received, after a period of washout, $5 \mathrm{mg} \alpha$-tocopherol. IV, intravenous; PO, per oral administration.

Effect of liver fat on kinetics parameters of $d_{\sigma}-\alpha$-tocopherol in healthy versus $H S$ subjects. Based on the reduced $\mathrm{d}_{6}$ - $\alpha$-tocopherol $\mathrm{C}_{\max }$ and AUC suggesting decreased tocopherol output from the liver, we separately characterized $d_{6}-\alpha$-tocopherol release kinetics in healthy and HS subjects over the first 4 hours, when release was linear (initial rate conditions, Figure $3 \mathrm{~B}$ ). Although the rate of removal of $\mathrm{d}_{6}-\alpha$-tocopherol (disappearance) from plasma into the liver was similar in healthy and HS subjects $(P>0.49$; Supplemental Figure 1$)$, the rate of $d_{6}-\alpha$-tocopherol release from the liver into the circulation (reappearance) was reduced in HS versus healthy $2 \mathrm{mg}$ subjects $(0.057$ vs. $0.083 \mu \mathrm{M} / \mathrm{h}, P<0.001)$ (Figure 3, B and C). Upon quantitation of liver fat (measured by magnetic resonance spectroscopy, MRS; see Supplemental Methods), values of liver fat above $5 \%$ were associated with a reduced rate of $\mathrm{d}_{6}-\alpha$-tocopherol release (Figure $3 \mathrm{D}, R^{2}=0.62$ ). If body fat was also involved in $\mathrm{d}_{6}$ - $\alpha$-tocopherol sequestration, then body fat (determined by DEXA) should similarly be related to the rate of $d_{6}-\alpha$-tocopherol reappearance in plasma. We performed separate analyses on the HS cohort and the healthy cohort. No association was apparent in either healthy $\left(R^{2}=0.076\right.$, Figure $\left.3 \mathrm{E}\right)$ or HS groups $\left(R^{2}=0.007\right.$, Figure $\left.3 \mathrm{~F}\right)$. These data suggest that reduced tocopherol liver output in HS subjects is associated with reduced release kinetics.

Fat "traps" vitamin E in vitro and in vivo. We investigated in vitro and in animals whether fat increased $\alpha$-tocopherol content in the liver. When oleic acid fat-loaded hepatocytes were incubated with fluorescently labeled $\alpha$-tocopherol, they contained more tocopherol and lost less over time as assessed by flow cytometry and microscopy $(P<0.01$, Figure $4, \mathrm{~A}-\mathrm{C})$. Using oleic acid-loaded cells compared to controls, fluorescently labeled $\alpha$-tocopherol was colocalized to the fat droplet (Figure 4D and Supplemental Figure 2). Consistent with these observations, livers of mice fed a high-fat diet had more $\alpha$-tocopherol compared with controls, with content of $\alpha$-tocopherol in the diet held constant $(P<0.01$ and Figure 4E). These findings show that when hepatocytes are induced to accumulate fat intracellularly, the resulting lipid droplets sequester vitamin E. Taken together, these findings further support our hypothesis that excess liver fat sequesters $\alpha$-tocopherol, reducing output from the liver.

Plasma $d_{6}$ - and $d_{3}-\alpha$-tocopherol concentrations and redistribution into lipoproteins in 10 healthy versus 6 HS women: dual-isotope analyses. Just as $\mathrm{d}_{6}-\alpha$-tocopherol administered intravenously revealed liver physiology for $\alpha$-tocopherol, $d_{3}-\alpha$-tocopherol administered orally could be used to additionally evaluate intestinal physiology for $\alpha$-tocopherol. We compared pharmacokinetics and lipoprotein physiology in healthy 
A
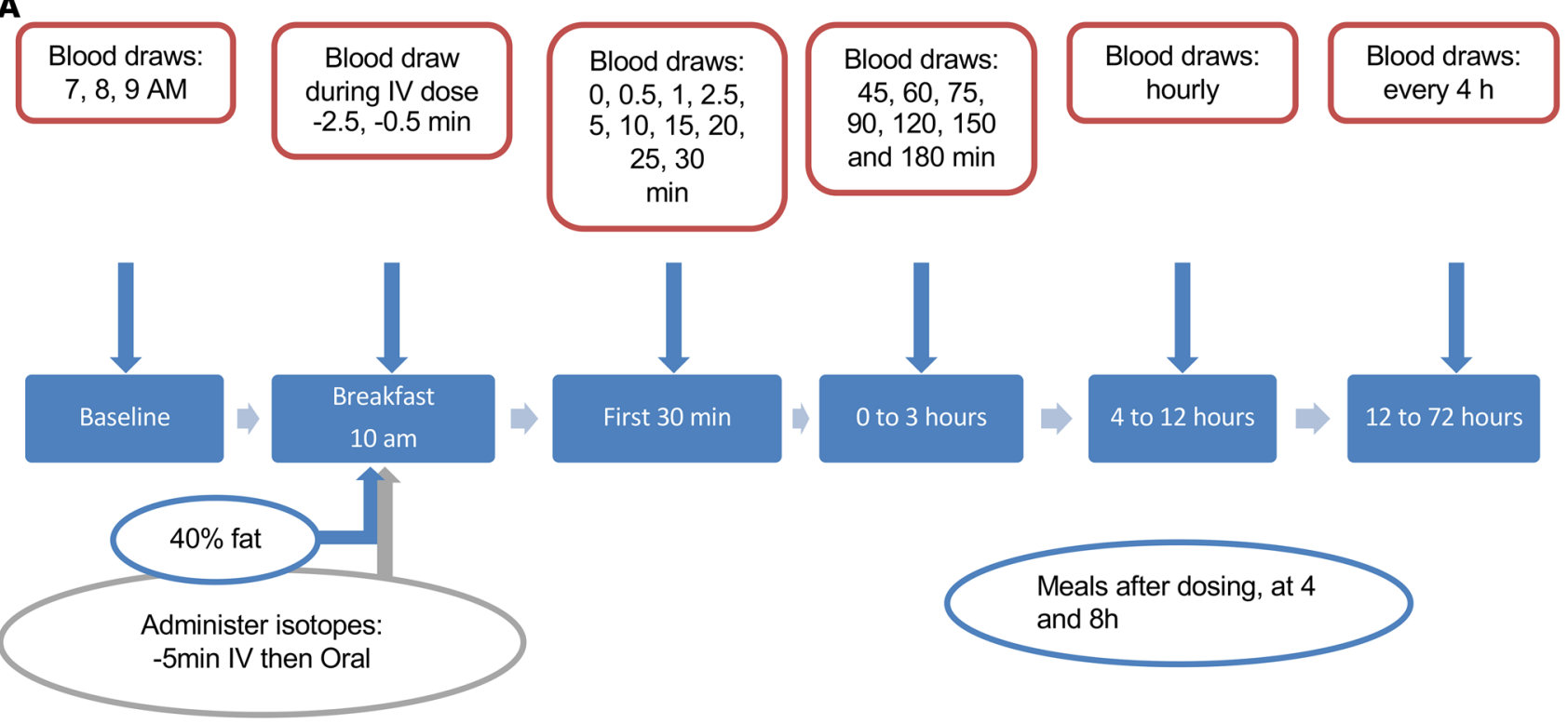

B

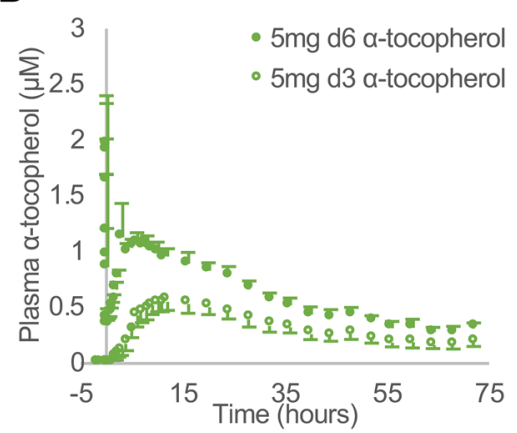

$\mathbf{E}$

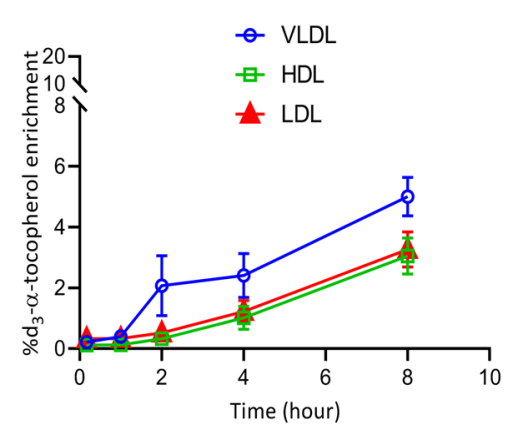

H

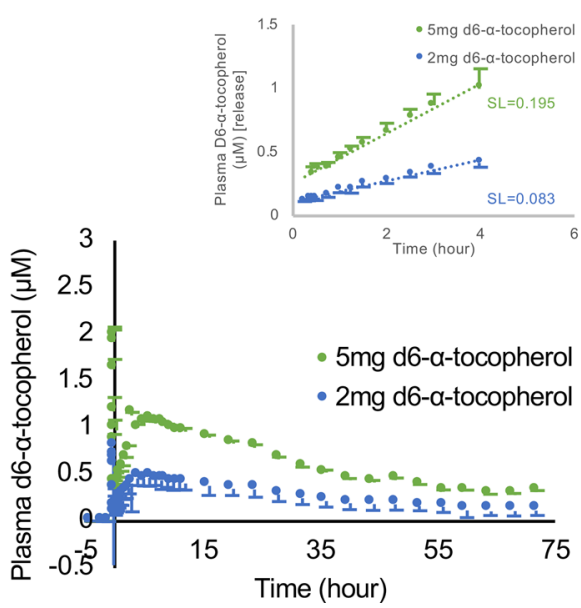

C

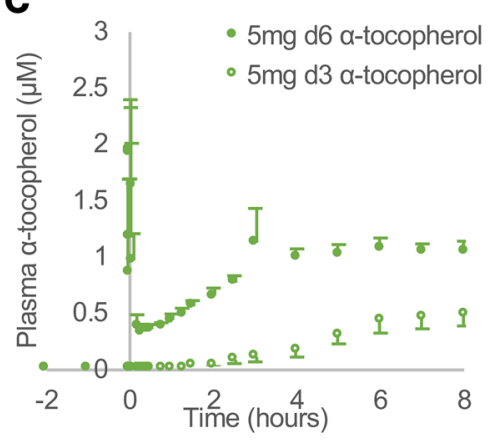

$\mathbf{F}$

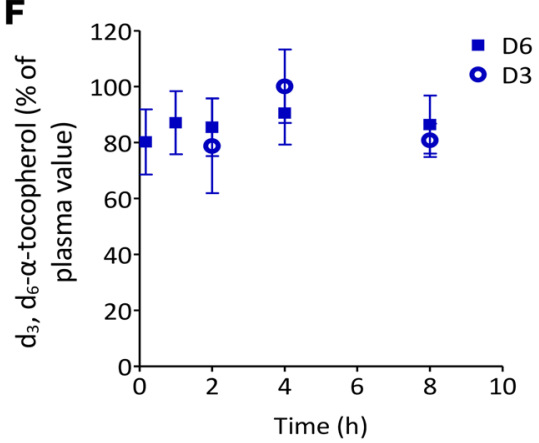

Meals after dosing, at 4 and $8 \mathrm{~h}$
D
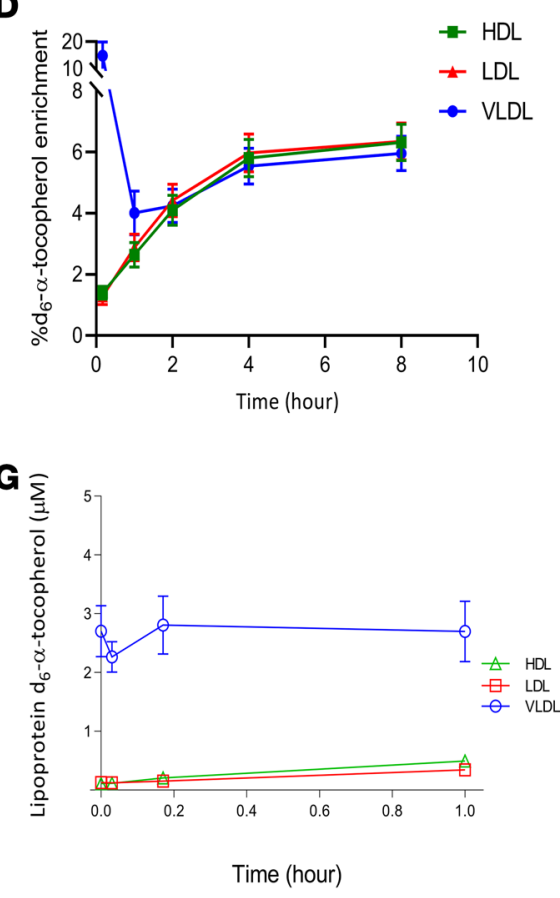

Figure 2. Plasma concentrations of $d_{6}-$ and $d_{3}-\alpha$-tocopherol and redistribution into lipoproteins in healthy women. (A) Protocol sampling scheme. (B) At time 0 in 6 subjects, $d_{5}-\alpha$-tocopherol $5 \mathrm{mg}(\bullet)$ was administered IV and $\mathrm{d}_{3}-\alpha$-tocopherol $5 \mathrm{mg}(\circ)$ was administered orally, with plasma samples obtained over 72 hours. (C) Plasma samples in B, magnified time interval -2 to 8 hours. (D) Percentage enrichment of $d_{6}-\alpha$-tocopherol ( $\triangle$, HDL; $\mathbf{\square}, \mathrm{LDL} ; \bullet, V L D L$ ) in lipoproteins following administration of $5 \mathrm{mg}$ at time 0 in 6 subjects. (E) Percentage enrichment of $d_{3}$ - $\alpha$-tocopherol ( $\left.\triangle, H D L ; ~ \square, ~ L D L ; ~ O, V L D L\right)$ in lipoproteins following administration of $5 \mathrm{mg}$ at time 0 in 6 subjects. Because HDL and LDL points were superimposed, for clarity HDL and LDL curves were slightly offset on the $y$ axis. (F) Percentage of recovery of $d_{3}-\alpha$-tocopherol ( $(\circ)$ and $d_{6}-\alpha$-tocopherol $(\square)$ in all lipoproteins compared with plasma concentrations in 6 subjects. (G) Distribution of $7 \mu \mathrm{M}$ of $d_{5}-\alpha$-tocopherol in lipoproteins over time ex vivo $(\Delta, H D L$; $\square, L D L ; ~ \circ$, VLDL). (H) At time 0 in 6 subjects, $d_{5}-\alpha$-tocopherol $5 \mathrm{mg}(\bullet)$ and $2 \mathrm{mg}(\bullet)$ was administered IV, with plasma samples obtained over 72 hours; inset: initial rates of $d_{5}-\alpha$-tocopherol reappearance for these 2 doses. SL, slope: $0.195 \mu \mathrm{M} / \mathrm{h}$ for 5 -mg dose and $0.083 \mu \mathrm{M} / \mathrm{h}$ for 2 -mg dose. Data represented as mean \pm SEM. $n=6$ for HS and $n=10$ for healthy cohorts. 
A
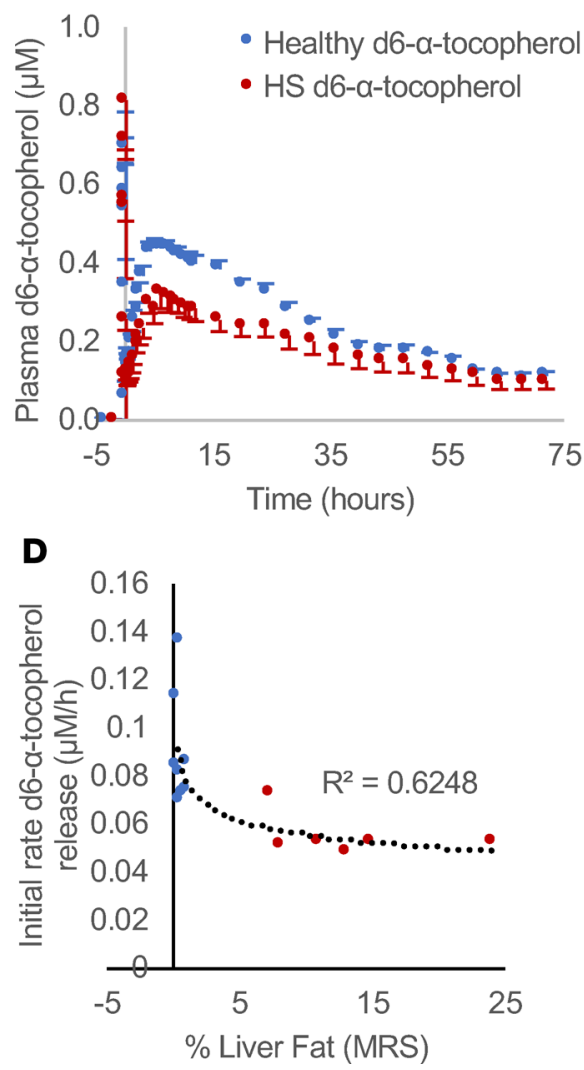

$\mathbf{B}$
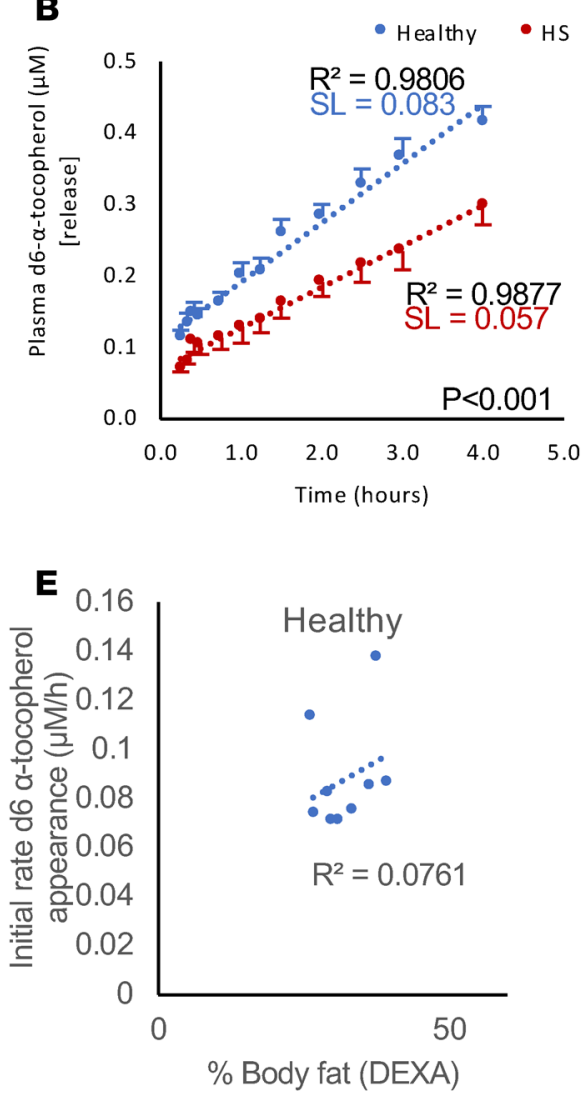

C

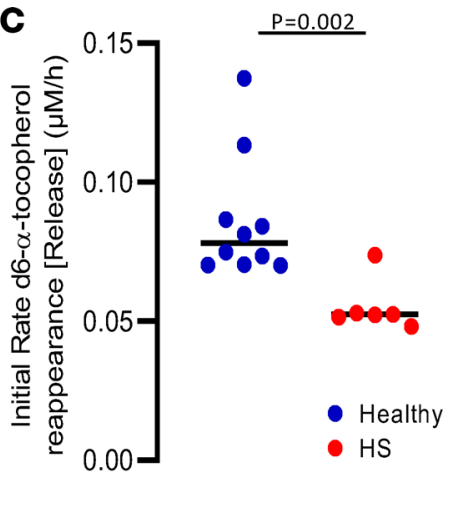

$\mathbf{F}$

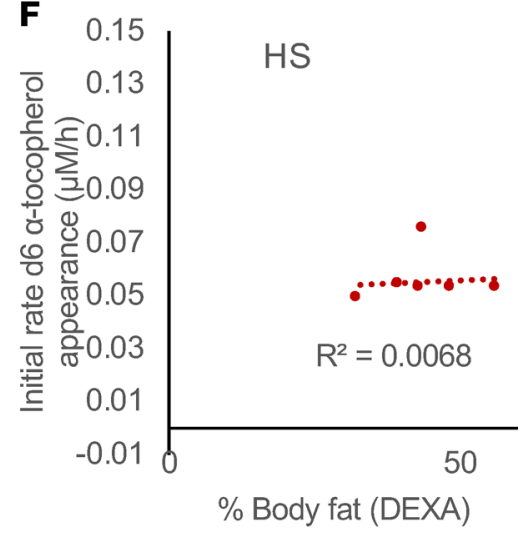

Figure 3. Influence of liver fat on $d_{6}-\alpha$-tocopherol plasma concentrations: pharmacokinetics and kinetics in 10 healthy and 6 HS subjects. (A) $d_{6}-\alpha$-Tocopherol 2 mg administered IV in healthy $(\bullet)$ or HS subjects $(\bullet)$ over 72 hours. (B-D) Initial rates of $d_{6}-\alpha$-tocopherol reappearance matched to patient status (C), to percentage liver fat by MRI (D). (E and F) Initial rates of $d_{6}-\alpha$-tocopherol reappearance to percentage body fat by DEXA in healthy (E) and HS (F) subjects. $n=6$ for HS and $n=10$ for healthy cohorts. (A and B) Data represented as mean \pm SEM. (C) Data represented as median.

and HS subjects utilizing 2-mg dosing with both oral $d_{3}-\alpha$-tocopherol and intravenous $d_{6}-\alpha$-tocopherol (dual-isotope analyses).

Pharmacokinetics of $d_{3}-\alpha$-tocopherol in healthy versus $H S$ subjects. Concentrations of $d_{3}-\alpha$-tocopherol were measured in the same plasma samples as $d_{6}-\alpha$-tocopherol over 72 hours in healthy and HS subjects (Figure 5A). We compared bioavailability of $\mathrm{d}_{3}-\alpha$-tocopherol ( $\mathrm{AUC}_{0-72 \mathrm{~h}}$, relative bioavailability) and fractional absorption (AUC $\mathrm{d}_{3} / \mathrm{d}_{6}$, true bioavailability) in healthy and HS subjects (Table 2). There was a $20 \%$ reduction in relative bioavailability in HS subjects compared with healthy subjects ( 8.6 vs. $10.7 \mu \mathrm{M} \times \mathrm{h}$, AUC $_{0-72 h}$, Table 2). The fractional absorption was higher in HS subjects (67\%) compared with healthy subjects $(60.6 \%)$. Despite not achieving statistical significance, these differences raised the possibility that additional, extrahepatic factors might modulate $\alpha$-tocopherol pharmacokinetics.

Effects of liver and body fat on $d_{3^{-}}$and $d_{6}-\alpha$-tocopherol lipoprotein redistribution in healthy versus $H S$ subjects. Based on previous findings (Figure 2) that $\alpha$-tocopherol is localized to lipoproteins (VLDL, LDL, HDL) with liver-mediated $\alpha$-tocopherol lipoprotein redistribution, we calculated $d_{6}$ - and $d_{3}$ - $\alpha$-tocopherol percentage enrichment over 8 hours $\left(\mathrm{T}_{\max }\right)$ in healthy versus HS subjects. Because lipoprotein production is increased in subjects with fatty liver disease (24-27), we predicted that $\alpha$-tocopherol percentage enrichment would be reduced in the HS subjects. We compared the $A U C_{0-8 h}$ for $d_{6}$ and $d_{3}-\alpha$-tocopherol percentage enrichments in healthy versus HS patients (see Methods) and found significant reductions in both $\mathrm{d}_{6}$ - and $\mathrm{d}_{3}-\alpha$-tocopherol percentage enrichments $(P<0.05$ : Figure 5, B and C; Table 3; and Supplemental Figure 3). Reduced $\alpha$-tocopherol percentage enrichments in HS subjects' lipoproteins is consistent with the notion that liver fat sequesters vitamin $\mathrm{E}$, thereby altering $\alpha$-tocopherol physiology.

Increased VLDL production reflects increased lipoprotein production in fatty liver disease $(24,26)$. If only liver fat and no other body fat pools modulated $\mathrm{d}_{3}-\alpha$-tocopherol, then the degree of reduction in percentage enrichment for VLDL fractions should be similar for both the oral $d_{3}-\alpha$-tocopherol and intravenous 
Table 2. Pharmacokinetic parameters derived from the plasma following oral $d_{3}$ - $\alpha$-tocopherol and IV $d_{6}-\alpha$-tocopherol administration to healthy $(n=10)$ or HS $(n=6)$ women

\begin{tabular}{|c|c|c|c|c|c|}
\hline & & \multicolumn{2}{|c|}{ IV $d_{6}-\alpha$-tocopherol } & \multicolumn{2}{|c|}{ PO $d_{3}-\alpha$-tocopherol } \\
\hline Parameter & & & $P$ values & & $P$ values \\
\hline Elimination rate $\left(\mathrm{K}_{\mathrm{e}}\right)$ & Healthy & $0.024 \pm 0.001$ & & $0.022 \pm 0.001$ & \\
\hline \multirow[t]{2}{*}{ Half-life (h) } & Healthy & $30.0 \pm 1.4$ & & $31.5 \pm 1.3$ & \\
\hline & HS & $36.9 \pm 4.4$ & 0.181 & $39.0 \pm 5.2$ & 0.210 \\
\hline$C_{\max }(\mu M)^{A}$ & Healthy & $0.48 \pm 0.02$ & & $0.28 \pm 0.04$ & \\
\hline & HS & $7.5 \pm 0.7$ & 0.880 & $8.5 \pm 0.67$ & 0.011 \\
\hline \multirow[t]{2}{*}{ Fractional absorption, $0-72$ hours $^{c}$} & Healthy & & & $60.6 \% \pm 5.2 \%$ & \\
\hline & HS & & & $67.5 \% \pm 8.3 \%$ & 0.537 \\
\hline \multirow[t]{2}{*}{$\operatorname{AUC}_{0-72 h}(\mu \mathrm{M} \times \mathrm{h})^{c}$} & Healthy & $17.5 \pm 0.95$ & & $10.7 \pm 1.3$ & \\
\hline & HS & $13.2 \pm 1.84$ & 0.074 & $8.6 \pm 1.0$ & 0.212 \\
\hline
\end{tabular}

Student's $t$ test (unpaired, 2 tailed). ${ }^{A} C_{\text {max }}$, maximum concentration postnadir for the IV dose. ${ }^{B} T_{\text {max }}$, time of maximum concentration postnadir for the IV dose. ${ }^{\mathrm{C}}$ From the dual-isotope method: percentage absorption $=\left(\left[\right.\right.$ oral $\left.A U C_{0-72 h}\right] /\left[I_{V}\right.$ AUC $\left.\left.{ }_{0-72 h}\right]\right) \times 100 .{ }^{D} A U C_{0-72 h}$ is calculated from the plasma concentrations from 0 to 72 hours. $A \cup C_{0-8 h}$ is calculated from the plasma concentration from 0 to 8 hours corresponding to the time of $C_{\max }$ for $d_{6}$ - $\alpha$-tocopherol. $K_{e}$, constant of elimination.

$\mathrm{d}_{6}$ - $\alpha$-tocopherol in HS subjects. Conversely if another fat pool, such as intestinal fat, modulates primarily $d_{3}-\alpha$-tocopherol, then HS subjects might have a greater reduction of $d_{3}$ - $\alpha$-tocopherol in VLDL compared with $d_{6}-\alpha$-tocopherol. The data showed that the reduction in percentage lipoprotein enrichment at 8 hours in HS (vs. healthy subjects) for the $\mathrm{d}_{3}$ oral dose (Figure 5D, $P<0.01$ ) was 1.3 -fold higher than the reduction in percentage lipoprotein enrichment for the $\mathrm{d}_{6}$ IV dose (Figure 5E, $P<0.05$ ), implying that another fat pool could be involved.

We probed these findings in HS subjects by calculating $d_{3}-\alpha$-tocopherol bioavailability $\left(\mathrm{AUC}_{0-72 \mathrm{~h}}\right)$ as a function of body fat, with comparison with healthy subjects. Bioavailability of $d_{3}$ - $\alpha$-tocopherol was negatively correlated with body fat in HS subjects $\left(R^{2}=0.71\right.$, Figure $\left.5 \mathrm{~F}\right)$ but not in healthy subjects $\left(R^{2}=0.05\right.$, Figure $5 \mathrm{G}$ ). Because $\mathrm{d}_{3}$ - $\alpha$-tocopherol accounts for intestinal absorption, while $\mathrm{d}_{6}$ - $\alpha$-tocopherol does not, these findings imply that there is an additional role of intestinal fat in modulating $\alpha$-tocopherol physiology.

\section{Discussion}

Administration of intravenous $\mathrm{d}_{6}$ - $\alpha$-tocopherol revealed the roles of the liver in $\alpha$-tocopherol physiology in healthy women and its contribution to the vitamin's pathophysiology in women with HS. In healthy women, approximately $85 \%$ of $\mathrm{d}_{6}$ - $\alpha$-tocopherol administered intravenously was removed from the circulation within 20 minutes, followed by reappearance (release) in plasma and redistribution on lipoproteins. Redistribution was absent when blood was incubated with deuterated tocopherol ex vivo, indicating a role of the liver in $\alpha$-tocopherol physiology in humans. Use of both kinetics and pharmacokinetics approaches to characterize $\mathrm{d}_{6}$ - $\alpha$-tocopherol revealed profound differences between healthy and HS women. For pharmacokinetics, HS subjects had significantly reduced $\mathrm{C}_{\max }$ and AUC compared with $2 \mathrm{mg}$ healthy controls, consistent with reduced liver output of vitamin $\mathrm{E}$ despite administration of similar doses. Consistent with these data, a similar pattern of reduced $\mathrm{C}_{\max }$ and AUC was observed in $5 \mathrm{mg}$ versus $2 \mathrm{mg}$ healthy subjects (Supplemental Table 1). Combined with our cell and animal data, the altered pharmacokinetics strongly indicate that liver fat sequesters $\alpha$-tocopherol and attenuates its release, independent of the $\alpha$-tocopherol transfer protein. For kinetics, initial $\mathrm{d}_{6}$ - $\alpha$-tocopherol removal from the circulation (disappearance rate) was as rapid in women with HS as in healthy women, but the initial release kinetics of $d_{6}-\alpha$-tocopherol reappearance in plasma were reduced by $30 \%(P<0.001$; Figure 3B). A similar pattern of reduced release kinetics was seen when comparing $5 \mathrm{mg}$ versus $2 \mathrm{mg}$ in healthy subjects (Supplemental Figure 4). These data support the hypothesis that excess liver fat sequesters tocopherol and results in pathophysiology that is analogous to a reduced vitamin $\mathrm{E}$ dose in healthy subjects. Because triglyceride release rates are 2- to 3- fold higher in subjects with nonalcoholic fatty 

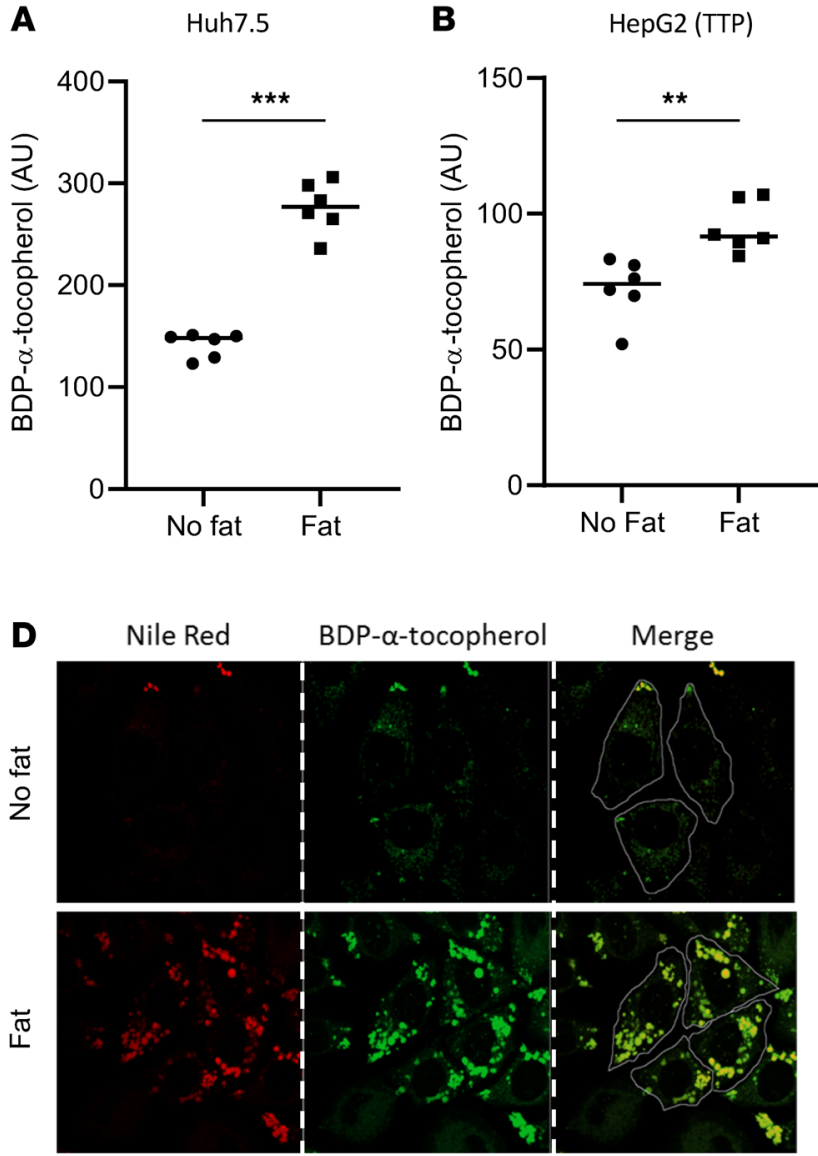

C

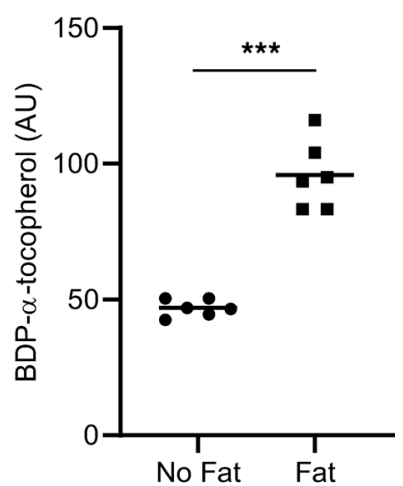

$\mathbf{E}$

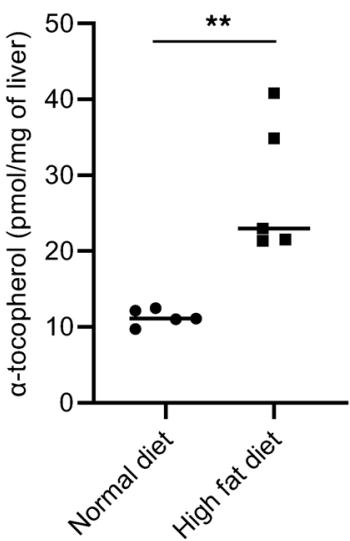

Figure 4. Fat traps vitamin $E$ in vitro and in vivo. (A-C) Bodipy- $\alpha$-tocopherol (BDP- $\alpha$-tocopherol) quantification by flow cytometry 30 hours after incubation using Huh7.5, HepG2C2A, or immortalized human hepatocyte (IHH) cells without/with $100 \mu \mathrm{M}$ oleic acid. $n=6$, representative experiment of 3 independent repeats. (D) Confocal microscopy of McARH7777 cells without/with $100 \mu$ M oleic acid (Nile Red staining) incubated 2 hours with $10 \mu \mathrm{M}$ BDP- $\alpha$-tocopherol (shown in green). Outlined areas indicate individual cell contours. (E) Mouse liver $\alpha$-tocopherol (HPLC analyses) using animals fed normal chow/high-fat normal vitamin $\mathrm{E}$ chow (HFNE); $n=5$. ${ }^{*} P<0.01$; and ${ }^{* * *} P<0.001$, Student's $t$ test (unpaired, 2 tailed). Data represented as mean \pm SD.

liver disease or metabolic syndrome (24, 26-28), our findings likely underestimate the actual sequestration of $\alpha$-tocopherol in livers of HS subjects. That $\mathrm{d}_{6}$ - $\alpha$-tocopherol sequestration is only associated with liver fat and is not affected by other fat stores (Figure 3, D and E) provides additional support of our hypothesis. Only with increasing liver fat was there a highly correlated inverse relationship to $d_{6}-\alpha$-tocopherol release.

Administration of oral $d_{3}$ - $\alpha$-tocopherol also revealed new physiology and pathophysiology, but findings with $\mathrm{d}_{3}$ - $\alpha$-tocopherol are more complex for several reasons. Several hours lapsed before $\mathrm{d}_{3}$ - $\alpha$-tocopherol appeared in plasma. $\alpha$-Tocopherol is first absorbed in intestine, undergoes lymphatic transport to the thoracic duct, and enters the general circulation before uptake by the liver and subsequent re-release (21-23). Consistent with other studies using only oral tocopherol (29), our findings revealed reduced $\mathrm{d}_{3}$ - $\alpha$-tocopherol bioavailability in $\mathrm{HS}$ versus healthy subjects, although the findings did not achieve statistical significance. Our data also revealed a negative correlation between $d_{3}-\alpha$-tocopherol bioavailability and percentage body fat (DEXA) but not liver fat. Taken together, our findings suggest that a body fat compartment, possibly intestinal fat, additionally modulates $\mathrm{d}_{3}$ - $\alpha$-tocopherol. The lack of statistical significance in $d_{3}$ - $\alpha$-tocopherol bioavailability for healthy versus HS subjects may be explained by the variability in percentage body fat in our cohort $(33 \%-57 \%)$ as well as effects of subsequent food ingestion on $\alpha$-tocopherol trafficking $(18,29)$. Future studies focusing on subjects with similar percentage of body fat, with and without HS, as well as controlled fasting and postprandial studies can address these possibilities. 
A

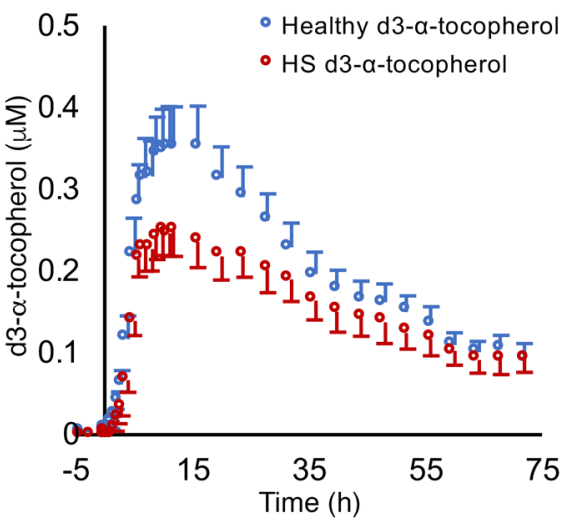

D
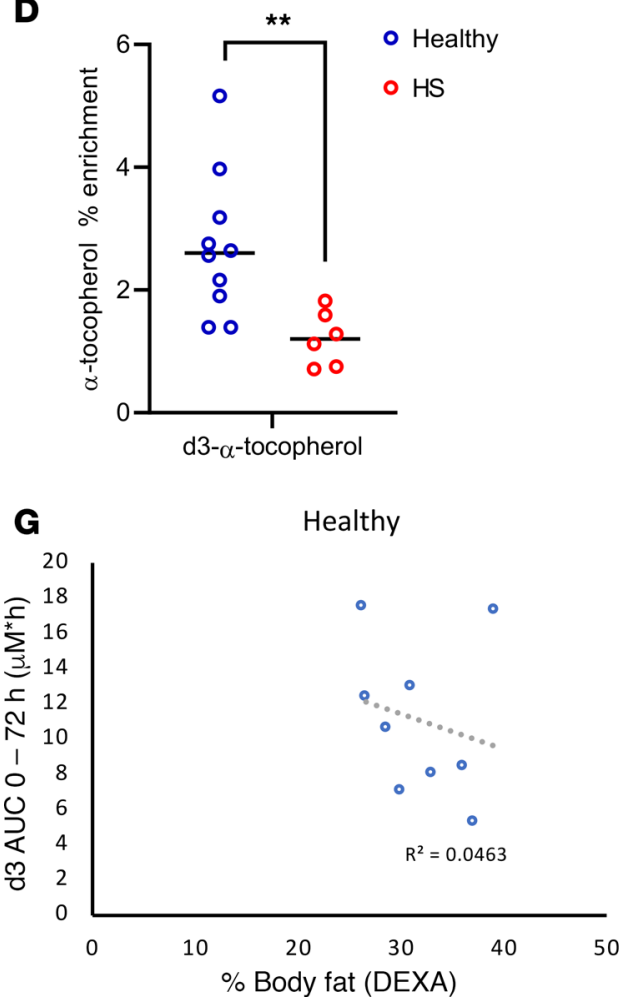

B
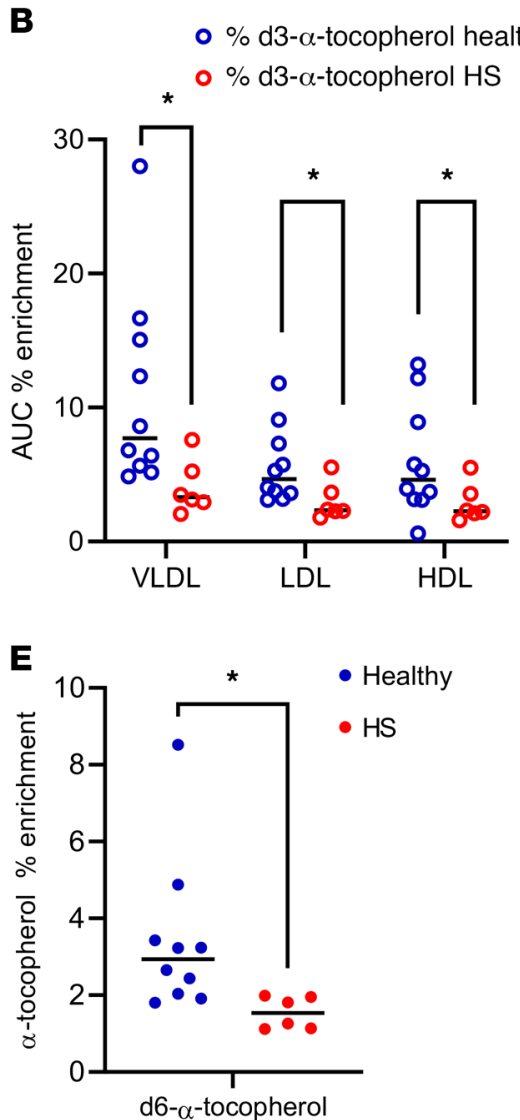

$\mathbf{F}$

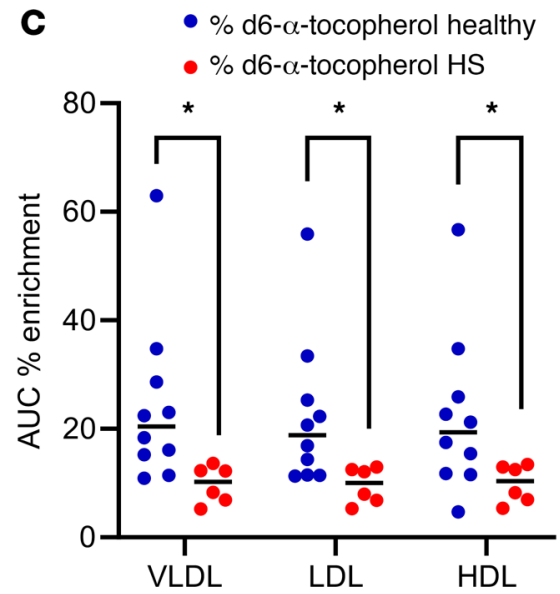

HS

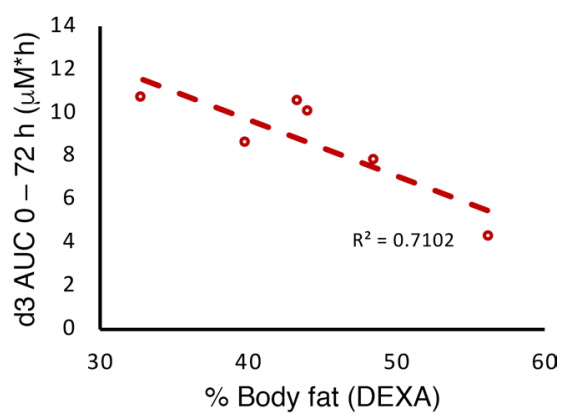

Figure 5. Plasma concentrations of $d_{6}$ - and $d_{3}-\alpha$-tocopherol and redistribution into lipoproteins in 10 healthy versus 6 HS women: dual-isotope analyses. (A) $d_{3}-\alpha$-Tocopherol 2 mg administered IV in healthy ( $($ ) or HS subjects ( $($ ) over 72 hours; data represented as mean \pm SD. (B and C) AUC of (B) $d_{3}$ - $\alpha$-tocopherol percentage enrichment and (C) $d_{6}-\alpha$-tocopherol percentage enrichment for VLDL, LDL, and HDL from 0 to 8 hours following administration of $2 \mathrm{mg} \mathrm{d}_{3}$ - or $\mathrm{d}_{6}$ - $\alpha$-tocopherol in healthy and HS subjects. (D and E) Percentage enrichment of (D) $d_{3}-\alpha$-tocopherol and (E) $d_{6}-\alpha$-tocopherol in VLDL in healthy $(\bullet)$ and HS $(\bullet)$ at time 8 hours; data represented as median. ${ }^{*} P<0.05$; ${ }^{*} P<0.01$, Student's $t$ test (unpaired, 2-tailed). (F and $\mathbf{G}) A U C_{0-72 h}$ of $d_{3}-\alpha$-tocopherol matched to percentage body fat by DEXA for HS (F) and healthy (G) subjects. $n=6$ for HS and $n=10$ for healthy cohorts.

To further evaluate the effect of body (i.e., intestinal) fat on $d_{3}-\alpha$-tocopherol physiology, we compared fractional absorption in HS versus healthy subjects, using the dual-isotope technique (18). Given the additional contribution that intestinal fat could have to modulate $d_{3}$ - $\alpha$-tocopherol but not $d_{6}-\alpha$-tocopherol, we had anticipated a reduction in the $\mathrm{d}_{3} / \mathrm{d}_{6}$ AUC ratio that would indicate reduced fractional absorption in the HS group. Surprisingly, our findings revealed higher fractional absorption in HS versus healthy subjects, albeit a statistically insignificant difference. These findings for fractional absorption in the HS group are likely due to relative changes in $d_{3}-\alpha$-tocopherol vis-à-vis changes in $d_{6}$ - $\alpha$-tocopherol, resulting from varying degrees of modulation by intestinal and liver fat. These findings also reveal limitations in the use of fractional absorption to estimate $\alpha$-tocopherol bioavailability in subjects who are obese or have hepatosteatosis. The findings also showed that both $d_{3}-$ and $d_{6}-\alpha$-tocopherol release on lipoproteins was reduced in women with HS compared with healthy subjects and that there were reductions in percentage enrichment of $d_{3}$-and $d_{6}$ - $\alpha$-tocopherol in several lipoprotein fractions in HS subjects, consistent with increased lipoprotein production associated with hepatosteatosis $(24,26,28)$. 
Table 3. AUC $C_{0-8 h}$ derived from lipoprotein percentage $d_{3}$ - $\alpha$-tocopherol and percentage $d_{6}-\alpha$-tocopherol enrichment

\begin{tabular}{|c|c|c|c|c|c|}
\hline \multicolumn{6}{|l|}{ Lipoprotein parameters } \\
\hline & & $d_{6}-\alpha$-tocopherol & $P$ values & $d_{3}-\alpha$-tocopherol & $P$ values \\
\hline VLDL \% enrichment, AUC (\% / h) & Healthy & $24.4 \pm 4.9$ & & $11.0 \pm 2.3$ & \\
\hline \multirow[t]{2}{*}{ LDL \% enrichment, AUC (\% / h) } & Healthy & $22.3 \pm 4.4$ & & $6.3 \pm 1.2$ & \\
\hline & HS & $9.6 \pm 1.0$ & 0.019 & $3.0 \pm 0.4$ & 0.027 \\
\hline HDL \% enrichment, AUC (\% / h) & Healthy & $22.2 \pm 4.7$ & & $6.0 \pm 1.3$ & \\
\hline
\end{tabular}

$A U C_{0-8 \mathrm{~h}}$ is calculated from the lipoprotein percentage enrichment (mean $\pm \mathrm{SEM}$ ), as described in Methods, following $2 \mathrm{mg} I V \mathrm{~d}_{6}-\alpha$-tocopherol administration in 10 healthy subjects and 6 HS subjects. Student's $t$ test (unpaired, 2 tailed).

The approximately 1.3 -fold reduction in $d_{3}$ versus $d_{6}$ percentage enrichment in HS subjects was consistent with an added role of a body fat compartment (i.e., intestinal fat) in $d_{3}$ - $\alpha$-tocopherol modulation. Sequestration of vitamin $\mathrm{E}$ in other body locations with excessive fat pools associated with obesity, such as an intestinal fat pool, might further reduce vitamin E delivery to the liver. Downstream potential consequences of extrahepatic fat sequestration sites include reduction in bioavailable intrahepatic vitamin $\mathrm{E}$ and reduced antioxidant capacity. To test consequences of excess body fat pools, future studies can investigate $\mathrm{d}_{6}-\alpha$-tocopherol and $\mathrm{d}_{3}-\alpha$-tocopherol kinetics in obese patients who do not have hepatosteatosis.

Subjects in this study were selected based on percentage HS and specifically chosen to have less than $2 \%$ or more than $7 \%$ steatosis, so as to bookend potential effects of liver fat. Women were purposely selected to have steatosis but not hepatitis, based on normal-range serial liver function testing and FIB- 4 score (30). Although liver biopsy is the gold standard to diagnose hepatitis, we were unable to justify the risk of biopsy given our inclusion criteria and absence of patient-specific benefit that could be expected from biopsy versus risk of harm.

It is unknown whether sequestered vitamin $\mathrm{E}$ in humans is functional, quiescent, or used by increased antioxidant requirements. In the liver, it is possible that sequestered vitamin $\mathrm{E}$ might be used to quench oxidants generated within excess fat. Nevertheless, because of sequestration of vitamin E, there may be less of it available to other cellular compartments. As a consequence of antioxidant crosstalk, discussed in the next paragraph, less tocopherol availability could increase risk of oxidant damage in many hepatocellular compartments.

Based on its established activity as an antioxidant (electron donor) (31-34), $\alpha$-tocopherol may be used for quenching oxidants that are generated during hepatic cell metabolism, especially as an outcome of lipid oxidation $(35,36)$. Hydrophobicity of vitamin E confines its direct antioxidant activity to the lipid milieu of biological membranes, distinct from the redox status in water-soluble compartments (37). The cellular $\mathrm{NAD}(\mathrm{P}) \mathrm{H} /$ glutathione antioxidant system is reserved mostly for soluble targets and for reduction of peroxidative end products - lipid hydroperoxides. Loss of glutathione-dependent reductases (for instance the selenium-dependent glutathione peroxidase 4) substantially raises oxidative events and stress to cells. Much of this can be avoided by lipid-soluble antioxidants, such as $\alpha$-tocopherol, which limit production of lipid peroxides by terminating the radical chain reaction (34). Additionally, there is in vitro evidence that interaction, or crosstalk, occurs between water- and lipid-soluble antioxidant molecules at the membrane-cytosol interface $(38,39)$, along with support from clinical data (40). Thus, via direct and indirect actions, vitamin $\mathrm{E}$ can have antioxidant activity in multiple cell compartments. Although other $\alpha$-tocopherol functions in the liver are poorly characterized, $\alpha$-tocopherol could influence membrane transport of nutrients into hepatocytes, intermediary metabolism of lipids, activities of membrane-associated enzymes in sugar catabolic pathways, or lipid export $(41,42)$.

What is the clinical relevance of $\alpha$-tocopherol sequestration and decreased hepatic release? The data suggest that when obesity is accompanied by liver fat, meal-derived $\alpha$-tocopherol as well as lipoprotein-derived $\alpha$-tocopherol could be diverted to a liver fat depot, perhaps with diminished local availability. Especially after food ingestion, fat in hepatocytes might generate excess oxidants $(5,25,43)$, precisely when there would be decreased availability of $\alpha$-tocopherol for oxidant quenching, perhaps leading to chronic oxidant-induced liver damage (i.e., progression to inflammation, hepatocyte injury, and irreversible cirrhosis). In an obese individual, this chain of events is repeated over thousands of 


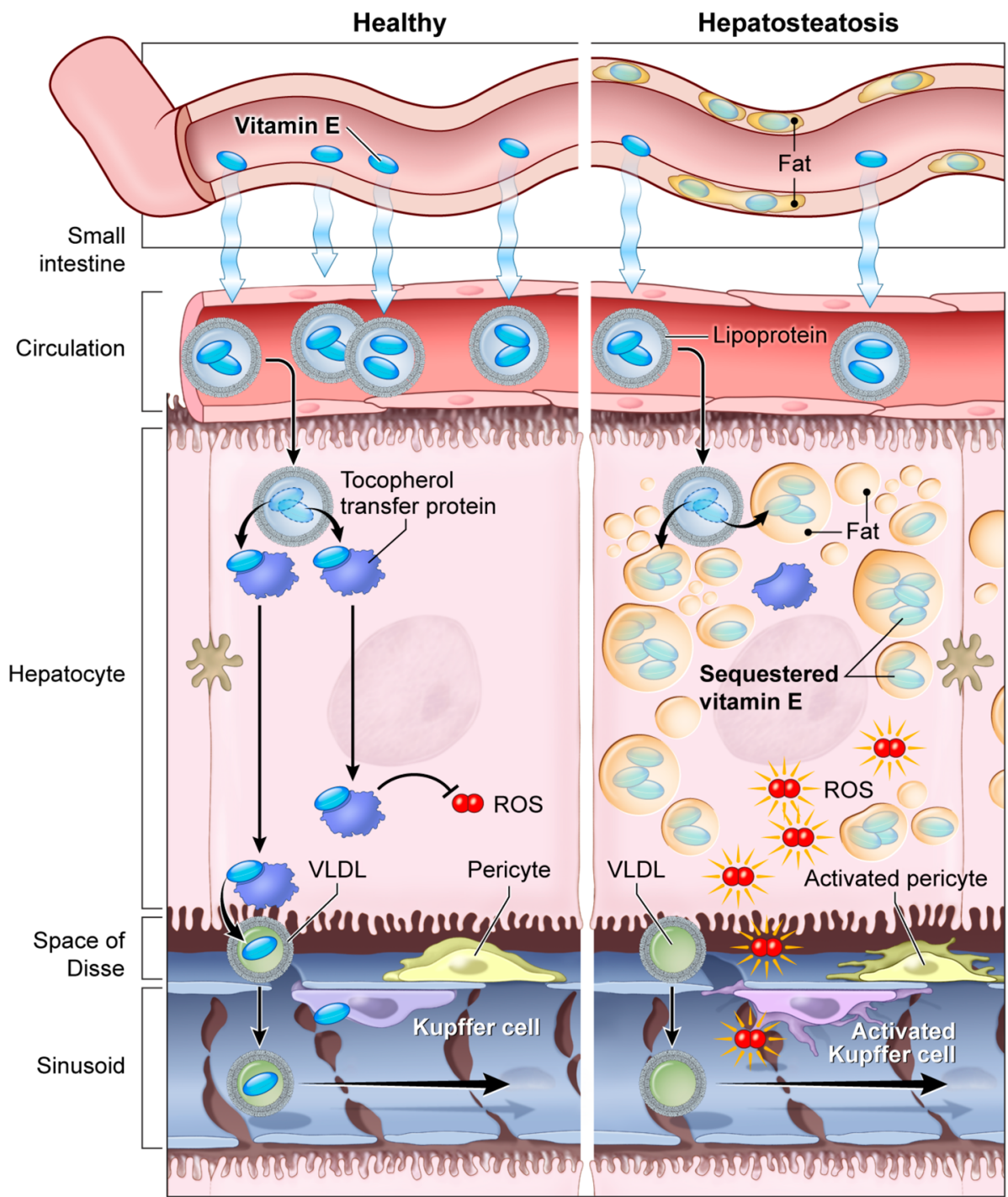

Figure 6. Proposed vitamin E ( $\alpha$-tocopherol) physiology in healthy subjects and pathophysiology in subjects with HS. Left (healthy subjects): Following oral ingestion, $\alpha$-tocopherol is transported (via chylomicrons to the thoracic duct and general circulation) to hepatocytes on lipoproteins. Internalized $\alpha$-tocopherol is specifically recognized and transported through hepatocytes by tocopherol transfer protein (TTP), with release into the space of Disse and lipoprotein capture, shown as VLDL. Biologically available vitamin E quenches reactive oxygen species (ROS) generated by normal hepatocyte metabolism. Right (subjects with HS): Following oral ingestion, some $\alpha$-tocopherol might be sequestered in intestinal fat (i.e., extrahepatic fat) with a reduction in availability. Remaining available vitamin $\mathrm{E}$ is transported to hepatocytes on lipoproteins. Internalized $\alpha$-tocopherol is diverted into liver fat, acting as a chemical sink, resulting in decreased vitamin $E$ availability within the hepatocyte and a functional local hepatocyte deficiency. Additionally, vitamin E local release into the space of Disse may be reduced. Less local vitamin E could lead to unquenched ROS that can damage hepatocytes directly and/or activate pericytes and Kupffer cells, over time producing inflammation, hepatitis, and fibrosis (cirrhosis).

meals, leading to further exacerbation of NASH. Overall, it is possible that excess food intake may inadvertently create a local vitamin deficiency because of unintended fat deposition in liver: famine from feast (refs. 44, 45 and Figure 6).

The famine-from-feast concept opens unexpected experimental and treatment doors. For NASH, the concept supplies a reason why only some patients respond to treatment with vitamin $\mathrm{E}$ ( $\alpha$-tocopherol) $(7,8)$. By the time hepatitis is clinically apparent, correcting local chronic vitamin $\mathrm{E}$ deficiency may be too late, with irreversible fibrosis already present. Although there are clues (46), it is unknown whether liver fat affects disposition and metabolism of the 3 other fat-soluble vitamins in humans, and vitamin E experiments here may serve as a paradigm. Rodents have a different lipoprotein profile than humans (47-49), and serial sampling to detect rapid 
hepatic uptake and release in rodents is not possible with current technology. Famine from feast derives from nutrition concepts advanced more than 70 years ago, concepts that disease could increase vitamin requirements $(50,51)$. Knowledge and tools did not exist then to explore how nutritional needs are changed by disease but do now. As exemplified by vitamin $\mathrm{C}$ and data presented here, characterization of normal physiology and pharmacokinetics of vitamins in humans may unveil pathophysiology plus unexpected use in disease treatment $(44,52-54)$. From the data here, it is possible that a fat-induced vitamin deficiency may be correctable: increased vitamin $\mathrm{E}$ ingestion could restore a normal hepatic release pattern. A path already exists for vitamin supplementation to prevent disease. Folate addition to flour is estimated to be responsible for a $30 \%$ decrease of neural tube defects in the United States (55). The data here raise the intriguing possibility that timely vitamin E supplementation might attenuate progression of HS to NASH, perhaps by correcting fat-induced, localized, hepatic vitamin E deficiency before inflammation, hepatitis, and fibrosis. We believe that studies to advance these possibilities should proceed expeditiously.

\section{Methods}

\section{Materials}

BDP $\alpha$-tocopherol was synthesized as described previously (19). Propidium iodide (PI), cell culture media, and reagents for cell culture were purchased from Life Technologies. HepG2 and McARH7777 (rat hepatoma; ATCC CRL-1601) cells were purchased from ATCC. The Huh7.5 (hepatocellular carcinoma) cell line was provided by Francis Chisari (The Scripps Research Institute, La Jolla, California, USA). Cells expressing TTP were previously described (12). IHHs were a gift from R. Ray, St. Louis University, St. Louis, Missouri, USA. Unless specified, all chemicals were purchased from MilliporeSigma; solvents were purchased from Thermo Fisher Scientific.

\section{Clinical research trial}

Patient screening and enrollment. Clinical research to study $\alpha$-tocopherol pharmacokinetics in women was approved by the Institutional Review Board of National Institute of Diabetes and Digestive and Kidney Diseases and National Institute of Arthritis and Musculoskeletal and Skin Diseases, NIH, and conducted in accordance with NIH guidelines (protocol 09-DK-0097). This trial, a nonrandomized, nonblinded cohort and crossover intervention, was registered at ClinicalTrials.gov as NCT00862433. Healthy women (aged 18-40 years) with normal physical exams, screening laboratory studies, and liver fat less than $2 \%$ based on MRS and without serious or chronic medical illnesses were eligible. Women with HS (aged 18-55) were eligible if they had liver fat at least 7\% (measured by MRS); had no other causes of chronic liver disease, such as autoimmune, alcoholic, or infectious hepatitis; and had no other uncontrolled medical illness (blood pressure $<160 / 90 \mathrm{mmHg}$; hematocrit $>30 \%$; HgbAlc $<8.5 \%$; and absence of significant microvascular or macrovascular diabetic complications). Exclusion criteria included active tobacco use; alcohol abuse (estimated average alcohol consumption $>20 \mathrm{~g} / \mathrm{d}$ in the 6 months before enrollment or binge-drinking behavior); recent illicit drug use; food allergy to soy, egg, milk protein (casein), or wheat/gluten; coagulopathy; and unwillingness to use effective contraceptive methods for the duration of the study. All women studied had serial normal liver function tests, normal platelet counts, and FIB-4 scores less than 0.92 (ref. 30 and Table 1).

We screened 39 subjects and enrolled 10 healthy and 6 HS subjects who met inclusion criteria. Among the healthy group, we used 2 doses ( $5 \mathrm{mg}$ and $2 \mathrm{mg}$ ) of deuterated tocopherols. All 10 healthy subjects participated in the 2-mg intervention, while 6 out of the 10 also participated in the 5 -mg intervention. Half-lives for $d_{6}$ - and $d_{3}$ - $\alpha$-tocopherol were less than 40 hours (18), and the washout period used was more than 1400 hours ( $>2$ months; $>30 \times$ half-life) between interventions. All HS subjects received the 2-mg intervention.

Deuterated $\alpha$-tocopherol administration and sampling in humans. $\mathrm{d}_{3}-\alpha$-Tocopherol and $\mathrm{d}_{6}$ - $\alpha$-tocopherol were synthesized as described previously $(15,16,20)$ under good manufacturing practices (GMP). $d_{3}-\alpha$-Tocopherol for oral administration was diluted in tocopherol-stripped soybean oil at a concentration of $50 \mathrm{mg} / \mathrm{mL}$. Stripping is a commercial process in which natural oils are distilled at $250^{\circ} \mathrm{C}$ under vacuum to remove volatile phenols, such as vitamin E. Although the detailed protocol is proprietary, examples of the process's efficiency are documented $(56,57)$. For the $d_{6}$ - $\alpha$-tocopherol, no additives were added, and it was prepared for intravenous administration as a sterile lipid emulsion under GMP as described (17). The oral and intravenous preparations were administered to patients under an IND application. 
Enrolled subjects were admitted to the NIH Clinical Research Center as inpatients for 4-5 days. Subjects were fasted overnight from day 0 to day 1 . On day 1 early morning intravenous cannulae were inserted into both arms (see Figure 2A for protocol sampling scheme). For the morning meal, all subjects received the same custom-prepared liquid meal containing $40 \%$ fat. When two-thirds of the liquid meal was consumed, $\mathrm{d}_{3}$ - $\alpha$-tocopherol (the oral vitamin $\mathrm{E}$ dose at either $2 \mathrm{mg}$ or $5 \mathrm{mg}$ ) was placed on the subject's tongue, and the remainder of the liquid meal was consumed within 5 minutes. At that time, the intravenous $\mathrm{d}_{6}$ - $\alpha$-tocopherol dose (the same as the oral dose) was administered in the opposite arm of the sampling arm, with the infusion end defined as time 0 . Blood samples were obtained as shown in the protocol sampling scheme (Figure 2A). Samples were placed on ice at the bedside before plasma isolation by centrifugation, aliquoting into cryovials, flash-freezing in dry ice, and storing at $-80^{\circ} \mathrm{C}$ until analyses.

Dietary and nutritional monitoring. All consumed food was prepared by the NIH nutrition staff using tocopherol-stripped vegetable oil. As for diet composition, subjects' meals, snacks, and beverages contained minimal amounts of vitamin $\mathrm{E}$ (on average, $<6 \mathrm{mg} \alpha$-tocopherol/d and $\sim 12 \mathrm{mg} \gamma$-tocopherol/d). Diets were composed of $55 \%$ carbohydrate, $15 \%$ protein, and $30 \%$ fat. Food items rich in vitamin E were excluded (such as wheat germ oil, almonds, sunflower seeds, snack chips, and spinach). To avoid confounding results with variable vitamin $C$ status $(31,58)$, ascorbic acid stores were saturated in all subjects by prescribing $1 \mathrm{~g}$ oral vitamin $\mathrm{C}$ daily for 7 days starting 2 weeks before admission, followed by $250 \mathrm{mg}$ daily beginning 1 week before admission and continuing for the duration of the subject's participation in the study.

Imaging to quantitate liver and body fat. Liver fat was determined by MRS, performed on 3T MRI scanners (Achieva, Philips). Body fat was determined by DEXA whole-body composition analyses (Hologic Discovery QDR).

\section{Analytical procedures and statistics}

Lipoprotein isolation. Plasma samples were obtained after infusion of $\mathrm{d}_{6}$ - $\alpha$-tocopherol at times $0,0.17,1,2$, 4 , and 8 hours. Lipoproteins (VLDL, LDL, HDL) were separated using discontinuous salt density gradient ultracentrifugation as described previously (59). For lipoprotein isolation using ex vivo blood samples, 30 minutes before administration of the $d_{6}$ and $d_{3}$ - $\alpha$-tocopherols to subjects, four $10-\mathrm{mL}$ blood samples from each subject were withdrawn directly into sodium heparin tubes. To produce a final concentration of $7 \mu \mathrm{M}$, $5.5 \mu \mathrm{L}$ of $\mathrm{d}_{6}-\alpha$-tocopherol intravenous injection solution (concentration: $5.4 \mu \mathrm{g} / \mu \mathrm{L}$ ) was added ex vivo to each $10-\mathrm{mL}$ blood sample, followed by incubation at $37^{\circ} \mathrm{C}$ for indicated times of $0,2,10$, and 60 minutes. At the end of incubation, samples were processed for lipoprotein separation by ultracentrifugation as described (59).

$\alpha$-Tocopherol analyses. Labeled $\mathrm{d}_{3^{-}}, \mathrm{d}_{6^{-}}$, and $\mathrm{d}_{9}$ - $\alpha$-tocopherol $\left(\mathrm{d}_{9}\right.$ as an internal standard) and unlabeled $\mathrm{d}_{0}-\alpha$-tocopherol were analyzed using high-performance liquid chromatography/mass spectrometry (LC/MS) in plasma and lipoproteins as described previously $(18,60,61)$. Samples were thawed at room temperature immediately before use and saponified at $70^{\circ} \mathrm{C}$ with alcoholic $\mathrm{KOH}$ and $1 \%$ ascorbic acid. Samples were then cooled, antioxidants ( $1 \%$ ascorbic acid in water and $25 \mu \mathrm{g}$ butylated hydroxytoluene in ethanol) and internal standards ( $d_{9}-\alpha$-tocopherol, Isotec Labs; the isotopic distribution was $0 \% \mathrm{~d}_{0}$ - $\alpha$-tocopherol, $0 \% \mathrm{~d}_{3}-\alpha$-tocopherol, $0 \% \mathrm{~d}_{6}-\alpha$-tocopherol, $0.6 \% \mathrm{~d}_{7}-\alpha$-tocopherol, $11.0 \% \mathrm{~d}_{8}$ - $\alpha$-tocopherol, and $88.4 \% \mathrm{~d}_{9}$ - $\alpha$-tocopherol; $>99 \%$ deuterium-labeled $\alpha$-tocopherol [ref. 62]) added, and samples extracted with hexane. The extracts were dried under nitrogen, resuspended in 1:1 ethanol/methanol, and then injected either into an LC/MS (Waters 2695 Separations Module and a Micromass ZQ2000) or an LC-MS/MS (either an Applied Biosystems/MDS Sciex API 3000 or a Waters XEVO TQD). For single-ion recording detection (negative mode), mass-to-charge ratio $(\mathrm{m} / \mathrm{z}$ ) data were obtained for $\alpha$-tocopherol ( $\mathrm{d}_{0}$ - $\alpha$-tocopherol, $m / z 429 ; \mathrm{d}_{3}$ - $\alpha$-tocopherol, $m / z 432 ; \mathrm{d}_{6}$ - $\alpha$-tocopherol, $m / z 435$; and $\mathrm{d}_{9}$ - $\alpha$-tocopherol, $\left.m / z 438\right)$ and $\mathrm{d}_{0}-\alpha$-tocopherol $(m / z 415)$. For MS/MS detection (negative mode), analytes were detected using multiple reaction monitoring of transitions: $\mathrm{d}_{0}$ - $\alpha$-tocopherol, $m / z 429 / 163 ; \mathrm{d}_{3}-\alpha$-tocopherol, $m / z$ 432/166; $\mathrm{d}_{6}$ - $\alpha$-tocopherol, $m / z$ 435/169; $\mathrm{d}_{9}$ - $\alpha$-tocopherol, $m / z 438 / 172$; and $\mathrm{d}_{0}$ - $\alpha$-tocopherol, $m / z$ 415/149.

Pharmacokinetics analyses. Plasma $\alpha$-tocopherol pharmacokinetics parameters were calculated for all subjects using concentration-time data following administration of $d_{6}$-and $d_{3}$ - $\alpha$-tocopherols. AUCs of plasma and lipoprotein $\mathrm{d}_{6}-\alpha$-tocopherols were calculated by using the trapezoidal rule. Commercially available software (Stata, www.stata.com) was used to estimate pharmacokinetics parameters (Table 2). To specifically evaluate the effect of liver fat on $\alpha$-tocopherol physiology, and to bypass confounding effects associated with gastrointestinal absorption, we compared pharmacokinetics parameters from intravenously administered $\mathrm{d}_{6}$ - $\alpha$-tocopherol: $\mathrm{C}_{\max }, \mathrm{T}_{\max }$, and $\mathrm{AUC}_{0-8 \mathrm{~h}}$. To account for intestinal fat that could potentially modulate $\alpha$-tocopherol physiology, we calculated similar pharmacokinetics parameters for the orally administered 
$\mathrm{d}_{3}$ - $\alpha$-tocopherol in both healthy and HS groups. Bioavailability for healthy and HS subjects was calculated as $d_{3}-\alpha$-tocopherol $A C_{0-72 h}$. $\alpha$-Tocopherol fractional absorption for 0-72 hours and 0-8 hours (absolute bioavailability) was calculated using these formulae: fractional absorption ${ }_{0-72 \mathrm{~h}}=\left(\left[\mathrm{d}_{3} \alpha \mathrm{t}\left(\mathrm{AUC}_{0-72 \mathrm{~h}}\right)\right] /\left[\mathrm{d}_{6} \alpha \mathrm{t}\right.\right.$ $\left(\right.$ AUC $\left.\left.\left._{0-72 \mathrm{~h}}\right)\right]\right) \times 100$, and fractional absorption ${ }_{0-8 \mathrm{~h}}=\left(\left[\mathrm{d}_{3} \alpha \mathrm{t}\left(\mathrm{AUC}_{0-8 \mathrm{~h}}\right)\right] /\left[\mathrm{d}_{6} \alpha \mathrm{t}\left(\mathrm{AUC}_{0-8 \mathrm{~h}}\right)\right]\right) \times 100$, where $\mathrm{d}_{3} \alpha \mathrm{t}$ represents $d_{3}-\alpha$-tocopherol and $d_{6} \alpha$ t represents $d_{6}-\alpha$-tocopherol. Pharmacokinetics calculations were separately calculated for $\mathrm{d}_{3}$ - and $\mathrm{d}_{6}$ - $\alpha$-tocopherol enrichment in lipoprotein fractions.

Kinetics analyses. Initial release kinetics of $\mathrm{d}_{6}$ - $\alpha$-tocopherol were calculated over the first 4 hours when release was linear. Calculation of $d_{3}$ - $\alpha$-tocopherol release kinetics was not possible because of insufficient sampling time points over the relevant period, because of constraints on patient blood volume withdrawal limits.

\section{Lipoprotein percentage enrichment calculations}

(1) $d_{3} \alpha \mathrm{t}_{(\text {sample corrected })}=\frac{d_{3} \alpha \mathrm{t}_{(\text {baseline area count })}}{d_{0} \alpha \mathrm{t}_{(\text {baseline area count })}} \times d_{0} \alpha \mathrm{t}_{(\text {baseline area count })}$

(2) $d_{6} \alpha \mathrm{t}_{(\text {sample corrected })}=\frac{d_{6} \alpha \mathrm{t}_{\text {(baseline area count })}}{d_{0} \alpha \mathrm{t}_{(\text {baseline area count })}} \times d_{0} \alpha \mathrm{t}_{(\text {baseline area count })}$

(3) total $\alpha \mathrm{t}=d_{3} \alpha \mathrm{t}_{(\text {sample corrected)(1) }}+d_{6} \alpha \mathrm{t}_{(\text {sample corrected)(2) }}+d_{0} \alpha \mathrm{t}_{(\text {sample })}$

(4) $d_{3} \alpha \mathrm{t} \%$ enrichment $=100 \times \frac{d_{3} \alpha \mathrm{t}_{(\text {sample corrected)(1) }}}{\text { total } \alpha \mathrm{t}(3)}$

(5) $d_{6} \alpha \mathrm{t} \%$ enrichment $=100 \times \frac{d_{6} \alpha \mathrm{t}_{(\text {sample corrected)(2) }}}{\text { total } \alpha \mathrm{t}(3)}$

$\mathrm{d}_{0} \alpha \mathrm{t}$, unlabeled $\alpha$-tocopherol.

\section{Cell and animal studies}

$B D P \alpha$-tocopherol experiments. Cells were loaded with $100 \mu \mathrm{M}$ of oleic acid (Avanti polar lipid) for 12 hours to 14 hours followed by incubation with BDP $\alpha$-tocopherol. After 2 hours' incubation cells were washed with culture media and then analyzed by flow cytometry $(T=0)$ or incubated with culture media with $1 \%$ FBS for 30 hours and then analyzed. After incubation cells were collected using trypsin, washed with PBS, stained with PI for 20 minutes, and analyzed by flow cytometry (ADP Cyan, Beckman Coulter), using FL-1 detector (emission: 488, excitation: 530/40) for BPD $\alpha$-tocopherol and FL-3 detector (emission: 488, excitation: 613/20) for PI. Only PI- cells (live cells) were used for BDP $\alpha$-tocopherol detection. The same quantity of cells was used in all measurements $\left(40,000 \mathrm{PI}^{-}\right.$cells). The mean of the total FL-1 fluorescence spectra was used to compare the difference in BDP $\alpha$-tocopherol quantity in different samples. For microscopy experiments with BDP $\alpha$-tocopherol, $\alpha$-tocopherol transfer protein expression was induced in McARH7777 cells with doxycycline for a 48-hour incubation (7). After 24 hours of induction, cells were loaded with $0 \mu \mathrm{M}$ or $100 \mu \mathrm{M}$ oleic acid for 24 hours. Cells were then loaded with BDP $\alpha$-tocopherol as above, and lipid droplets were stained with $100 \mathrm{nM}$ Nile Red. Cells were washed with PBS and fixed with 4\% paraformaldehyde for 15 minutes; coverslips were mounted on slides with Slow Fade and sealed. Images were taken using confocal microscopy (Zeiss LSM510Meta Confocal microscope; 100× objective; 488 argon laser, $543 \mathrm{HeNe}$ ) or fluorescence microscopy (EVOS Cell Imaging Systems, Life Technologies).

Animal experiments. Animal procedures were approved by the Animal Care and Use Committees at NIH and Case Western Reserve University. C57BL/6 mice, purchased from The Jackson Laboratory, were fed with normal chow or high-fat chow (high-fat/sucrose diet, $45 \%$ sucrose and $55 \%$ fructose $[w / v]$ ) with normal $\alpha$-tocopherol levels (Harlan Teklad custom diet TD.130142; $150 \mathrm{mg} / \mathrm{kg}$ diet of $\alpha$-tocopherol acetate). After 16 weeks on the diet, mice were starved overnight and sacrificed. To process samples, $30 \mathrm{mg}$ of mouse liver was homogenized (Turrax blender) into $100 \mu \mathrm{L}$ of cold PBS and diluted 1:1 using $100 \mu \mathrm{L}$ of ice-cold water. Ice-cold methanol $(300 \mu \mathrm{L})$ was added to the sample, which was vortexed for 1 minute, followed by addition of $600 \mu \mathrm{L}$ ice-cold hexane. Each sample was vortexed again for 1 minute, and $400 \mu \mathrm{L}$ of the upper phase was transferred to a new tube followed by drying (speedVac, Thermo Fisher Scientific). The dried material was resuspended into $50 \mu \mathrm{L}$ of ethanol (200 proof) and $0.5 \%$ butylated hydroxytoluene $(w / v)$. Ten microliters was used for HPLC analysis, and $\alpha$-tocopherol concentrations were normalized using liver weight. 
Cells and animal $\alpha$-tocopherol analyses. In cells and animal tissues, $\alpha$-tocopherol was analyzed by HPLC with coulometric electrochemical detection $(39,40)$ but with various modifications. The instruments were temperature-controlled autosampler and dual-piston pump (Waters Chromatography) and coulometric electrochemical detector (Coulochem III, ESA-Dionex). The detector settings were electrode 2, $600 \mathrm{mV}$; and electrode 1, $-400 \mathrm{mV}$. The mobile phase contained $30 \mathrm{mM}$ lithium acetate (final concentration) dissolved in $95 \%$ methanol/water, with flow rate of $1 \mathrm{~mL} / \mathrm{min}$ and injection volume of $10 \mu \mathrm{L}$. The column was silica-based $5 \mu \mathrm{m}, 4.6 \mathrm{~mm} \times 15 \mathrm{~cm}$ C-8 (Eclipse XDB-C8, Agilent Technologies). The column was conditioned with mobile phase at $1 \mathrm{~mL} / \mathrm{min}$ for $24-36$ hours before running standards and samples and washed once monthly with 30\% methanol/water for 24 hours, $1 \mathrm{~mL} / \mathrm{min}$. Guard Cartridges Bioadvantage Basic C8 $5 \mu \mathrm{m}$ were essential for optimum performance (Thomson Instruments) and were replaced after injection of 150-200 biological samples to avoid online sample oxidation.

\section{Statistics}

Statistical significance $(P<0.05)$ was analyzed as follows: 2-way ANOVA (with adjustment for post hoc multiple comparisons) for the data in Figure 3B. Student's $t$ test (unpaired, 2 tailed) was used for the data in Table 2 and in Figure 3C; Figure 4, A-C and E; and Figure 5, B-D. For the data of within-subject changes in $\mathrm{C}_{\max }$ and $\mathrm{AUC}_{0-72 \mathrm{~h}}$ following administration of intravenous $\mathrm{d}_{6}-\alpha$-tocopherol to 10 healthy subjects at $2 \mathrm{mg}$ and 6 healthy subjects at $5 \mathrm{mg}$, as shown in Supplemental Table 1, we used a Wald-type $F$ test of contrasts formed from regression coefficients in a mixed-effects model with Kenward-Roger approximation for the statistic's denominator degrees of freedom (63). ${ }^{*} P<0.05 ;{ }^{*} P$ $<0.01$; and ${ }^{* *} P<0.001 . n \geq 3$ for all in vitro experiments; for mouse experiment, $n=5$ per group; for all clinical experiments, $n$ indicated in figure legends, always $\geq 6$ subjects. The major statistical endpoints (primary objective) of the clinical study with healthy subjects and those with HS were mean differences in the $\mathrm{C}_{\max }$ and $\mathrm{AUC}_{0-8 \mathrm{~h}}$ (at time to $\mathrm{C}_{\max }$ or $\mathrm{T}_{\max }$ ) of $\mathrm{d}_{6}$ - $\alpha$-tocopherol. The study was originally designed to show a statistically significant $(P \leq 0.05)$ difference in $C_{\max }$ values of $50 \%$ or greater between the 2 groups with at least $90 \%$ power (18). Those design-stage power calculations were based on a standard deviation of 1.0 and indicated a minimum of 7 subjects per group; upon reviewing interim data, the estimated standard deviations were consistent with values much smaller than 1.0 for the primary objective's endpoint. Thus, enrollment concluded after enrolling 6 subjects in the HS group and 10 subjects in the healthy control group, and calculations were inverted to state power in terms of a detectable difference in $\mathrm{C}_{\max }$ values for a range of standard deviation values while targeting a conventionally acceptable level of power (minimum $80 \%$, up to $90 \%$ ), assuming such differences would be deemed statistically significant $(P \leq 0.05)$; see Supplemental Table 1.

Study approval. This study was reviewed and approved by the Institutional Review Board of the National Institute of Diabetes and Digestive and Kidney Diseases and National Institute of Arthritis and Musculoskeletal and Skin Diseases. Signed informed consent documents were obtained from each participant included in the study.

For the animal study, experimental protocols were approved by the Office of Animal Care and Use of NIH and National Institute of Diabetes and Digestive and Kidney Diseases at Bethesda, Maryland, USA.

\section{Author contributions}

RDS, DM, DWH, and ML conceived the study. MT, SJP, and ML developed methodology. ICE, PCV, YW, MN, SC, VT, LU, SS, HT, and ML investigated. JA, MG, and CYL acquired resources. PCV, ICE, $\mathrm{BH}, \mathrm{GB}$, and MT were responsible for data curating. ICE, PCV, and ML wrote the original draft. ICE, PCV, MGT, DM, RDS, and ML reviewed and edited the draft. MGT and ML acquired funding. ML supervised and held IND. KW was responsible for formal analysis.

\section{Acknowledgments}

The authors thank Jay Hoofnagle for thoughtful evaluation and suggestions, Monica Skarulis and Ahmed Gharib for clinical contributions, and the Intramural Research Programs at National Institute of Diabetes and Digestive and Kidney Diseases and National Heart, Lung, and Blood Institute; the NIH Metabolic Unit staff; National Institute of Diabetes and Digestive and Kidney Diseases Clinical Core staff; NIH Clinical Center Nutrition Staff; and Scott Leonard for technical assistance. 
This study was funded by grants DK053213-13 to ML, DK067494 to DM, and DK081761 to MGT; the Office of Dietary Supplements, Office of the Director, NIH; and DSM (Switzerland), with a grant to MGT to procure intravenous deuterated $\alpha$-tocopherol and instrumentation. Funders had no input into study outcomes.

Address correspondence to: Mark Levine, Molecular and Clinical Nutrition Section, Digestive Disease Branch, National Institute of Diabetes and Digestive and Kidney Diseases, NIH, Building 10, Room 4D52, 9000 Rockville Pike, 10 Center Drive, Bethesda, Maryland 20892, USA. Phone: 301.402.5588; Email: markL@nih.gov.

1. Cornier MA, et al. The metabolic syndrome. Endocr Rev. 2008;29(7):777-822.

2. Withrow D, Alter DA. The economic burden of obesity worldwide: a systematic review of the direct costs of obesity. Obes Rev 2011;12(2):131-141.

3. Kramer CK, Zinman B, Retnakaran R. Are metabolically healthy overweight and obesity benign conditions?: A systematic review and meta-analysis. Ann Intern Med. 2013;159(11):758-769.

4. Hardy T, Anstee QM, Day CP. Nonalcoholic fatty liver disease: new treatments. Curr Opin Gastroenterol. 2015;31(3):175-183

5. Suzuki A, Diehl AM. Nonalcoholic steatohepatitis. Annu Rev Med. 2017;68:85-98.

6. Diehl AM, Day C. Cause, pathogenesis, and treatment of nonalcoholic steatohepatitis. NEngl J Med. 2017;377(21):2063-2072

7. Sanyal AJ, et al. Pioglitazone, vitamin E, or placebo for nonalcoholic steatohepatitis. N Engl J Med. 2010;362(18):1675-1685.

8. Lavine JE, et al. Effect of vitamin E or metformin for treatment of nonalcoholic fatty liver disease in children and adolescents: the TONIC randomized controlled trial. JAMA. 2011;305(16):1659-1668.

9. Farrell GC, Larter CZ. Nonalcoholic fatty liver disease: from steatosis to cirrhosis. Hepatology. 2006;43(2 Suppl 1):S99-S112.

10. European Association for the Study of the Liver (EASL), European Association for the Study of Diabetes (EASD), European Association for the Study of Obesity (EASO). EASL-EASD-EASO Clinical Practice Guidelines for the management of non-alcoholic fatty liver disease. J Hepatol. 2016;64(6):1388-1402.

11. Manor D, Morley S. The alpha-tocopherol transfer protein. Vitam Horm. 2007;76:45-65.

12. Chung S, et al. Vitamin $\mathrm{E}$ and phosphoinositides regulate the intracellular localization of the hepatic $\alpha$-tocopherol transfer protein. J Biol Chem. 2016;291(33):17028-17039.

13. Harrison SA, Ramrakhiani S, Brunt EM, Anbari MA, Cortese C, Bacon BR. Orlistat in the treatment of NASH: a case series. Am J Gastroenterol. 2003;98(4):926-930.

14. Kawanaka M, et al. Treatment of nonalcoholic steatohepatitis with vitamins E and C: a pilot study. Hepat Med. 2013;5:11-16.

15. Ingold KU, Hughes L, Slaby M, Burton GG. Synthesis of 2R, 4'R, 8'R- $\alpha$-tocopherols selectively labelled with deuterium. J Labelled Comp Radiopharm. 1987;24(7):817-831.

16. Netscher T, Mazzini F, Jestin R. Tocopherols by hydride reduction of dialkylamino derivatives. Eur J Org Chem. 2007;7:1176-1183.

17. Lassnigg A, et al. Influence of intravenous vitamin $\mathrm{E}$ supplementation in cardiac surgery on oxidative stress: a double-blinded, randomized, controlled study. Br J Anaesth. 2003;90(2):148-154.

18. Traber MG, et al. Vitamin E absorption and kinetics in healthy women, as modulated by food and by fat, studied using 2 deuterium-labeled $\alpha$-tocopherols in a 3-phase crossover design. Am J Clin Nutr. 2019;110(5):1148-1167.

19. West R, Panagabko C, Atkinson J. Synthesis and characterization of BODIPY-alpha-tocopherol: a fluorescent form of vitamin E. J Org Chem. 2010;75(9):2883-2892.

20. Ghelfi M, Ulatowski L, Manor D, Atkinson J. Synthesis and characterization of a fluorescent probe for $\alpha$-tocopherol suitable for fluorescence microscopy. Bioorg Med Chem. 2016;24(12):2754-2761.

21. Forsgren L. Studies on the intestinal absorption of labelled fat-soluble vitamins (A, D, E, and K) via the thoracic-duct lymph in the absence of bile in man. Acta Chir Scand Suppl. 1969;399:1-29.

22. Drevon CA. Absorption, transport and metabolism of vitamin E. Free Radic Res Commun. 1991;14(4):229-246.

23. Traber MG. Vitamin E regulatory mechanisms. Annu Rev Nutr. 2007;27:347-362.

24. Fabbrini E, Mohammed BS, Magkos F, Korenblat KM, Patterson BW, Klein S. Alterations in adipose tissue and hepatic lipid kinetics in obese men and women with nonalcoholic fatty liver disease. Gastroenterology. 2008;134(2):424-431.

25. Fujita K, et al. Dysfunctional very-low-density lipoprotein synthesis and release is a key factor in nonalcoholic steatohepatitis pathogenesis. Hepatology. 2009;50(3):772-780.

26. Fabbrini E, et al. Intrahepatic fat, not visceral fat, is linked with metabolic complications of obesity. Proc Natl Acad Sci USA. 2009;106(36):15430-15435.

27. Shojaee-Moradie F, Ma Y, Lou S, Hovorka R, Umpleby AM. Prandial hypertriglyceridemia in metabolic syndrome is due to an overproduction of both chylomicron and VLDL triacylglycerol. Diabetes. 2013;62(12):4063-4069.

28. Adiels M, et al. Overproduction of large VLDL particles is driven by increased liver fat content in man. Diabetologia. 2006;49(4):755-765.

29. Mah E, et al. $\alpha$-Tocopherol bioavailability is lower in adults with metabolic syndrome regardless of dairy fat co-ingestion: a randomized, double-blind, crossover trial. Am J Clin Nutr. 2015;102(5):1070-1080.

30. Sterling RK, et al. Development of a simple noninvasive index to predict significant fibrosis in patients with $\mathrm{HIV} / \mathrm{HCV}$ coinfection. Hepatology. 2006;43(6):1317-1325.

31. Kagan VE, Serbinova EA, Forte T, Scita G, Packer L. Recycling of vitamin E in human low density lipoproteins. J Lipid Res. 1992;33(3):385-397.

32. Traber MG, Atkinson J. Vitamin E, antioxidant and nothing more. Free Radic Biol Med. 2007;43(1):4-15

33. Brigelius-Flohé R, Traber MG. Vitamin E: function and metabolism. FASEB J. 1999;13(10):1145-1155.

34. Maiorino M, Conrad M, Ursini F. GPx4, lipid peroxidation, and cell death: discoveries, rediscoveries, and open issues. Antioxid 
Redox Signal. 2018;29(1):61-74.

35. Chow CK, Ibrahim W, Wei Z, Chan AC. Vitamin E regulates mitochondrial hydrogen peroxide generation. Free Radic Biol Med. 1999;27(5-6):580-587.

36. Browning JD, Horton JD. Molecular mediators of hepatic steatosis and liver injury. J Clin Invest. 2004;114(2):147-152.

37. Burton GW, Joyce A, Ingold KU. Is vitamin E the only lipid-soluble, chain-breaking antioxidant in human blood plasma and erythrocyte membranes? Arch Biochem Biophys. 1983;221(1):281-290.

38. Shieh PK, Lanyi J, Packer L. Lipid-impregnated filter membranes: a stable planar membrane system for assay of electrical properties across reconstituted liposomes and natural membrane vesicles. Meth Enzymol. 1979;55:604-613.

39. Chan AC. Partners in defense, vitamin E and vitamin C. Can J Physiol Pharmacol. 1993;71(9):725-731.

40. Hamilton IM, Gilmore WS, Benzie IF, Mulholland CW, Strain JJ. Interactions between vitamins C and E in human subjects. Br J Nutr. 2000;84(3):261-267.

41. Softic S, et al. Divergent effects of glucose and fructose on hepatic lipogenesis and insulin signaling. J Clin Invest. 2017;127(11):4059-4074.

42. Gimenez MS, Oliveros LB, Gomez NN. Nutritional deficiencies and phospholipid metabolism. Int J Mol Sci. 2011;12(4):2408-2433.

43. Day CP, James OF. Steatohepatitis: a tale of two "hits"? Gastroenterology. 1998;114(4):842-845.

44. $\mathrm{Tu} \mathrm{H}$, et al. Low red blood cell vitamin $\mathrm{C}$ concentrations induce red blood cell fragility: a link to diabetes via glucose, glucose transporters, and dehydroascorbic acid. EBioMedicine. 2015;2(11):1735-1750.

45. Tu H, Wang Y, Li H, Brinster LR, Levine M. Chemical transport knockout for oxidized vitamin C, dehydroascorbic acid, reveals its functions in vivo. EBioMedicine. 2017;23:125-135.

46. Arai T, et al. Association of vitamin D levels and vitamin D-related gene polymorphisms with liver fibrosis in patients with biopsy-proven nonalcoholic fatty liver disease. Dig Liver Dis. 2019;51(7):1036-1042.

47. Behrens WA, Thompson JN, Madère R. Distribution of alpha-tocopherol in human plasma lipoproteins. Am J Clin Nutr. 1982;35(4):691-696.

48. Bjørneboe A, Bjørneboe GE, Bodd E, Hagen BF, Kveseth N, Drevon CA. Transport and distribution of alpha-tocopherol in lymph, serum and liver cells in rats. Biochim Biophys Acta. 1986;889(3):310-315.

49. Kostner GM, Oettl K, Jauhiainen M, Ehnholm C, Esterbauer H, Dieplinger H. Human plasma phospholipid transfer protein accelerates exchange/transfer of alpha-tocopherol between lipoproteins and cells. Biochem J. 1995;305(Pt 2):659-667.

50. Perla D, Marmorston J. The role of vitamin C in resistance. Arch Pathol. 1937;23:543-575.

51. Perla D, Marmortston J. The effect of vitamin C on resistence. In: Natural Resistance and Clinical Medicine. Boston, Masschusetts, USA: Little Brown \& Co.; 1941:1038-1091.

52. Levine M, et al. Vitamin C pharmacokinetics in healthy volunteers: evidence for a recommended dietary allowance. Proc Natl Acad Sci USA. 1996;93(8):3704-3709.

53. Levine M, Padayatty SJ, Espey MG. Vitamin C: a concentration-function approach yields pharmacology and therapeutic discoveries. Adv Nutr. 2011;2(2):78-88.

54. Shenoy N, Creagan E, Witzig T, Levine M. Ascorbic acid in cancer treatment: let the phoenix fly. Cancer Cell. 2018;34(5):700-706.

55. Williams J, et al. Updated estimates of neural tube defects prevented by mandatory folic acid fortification - United States, 19952011. MMWR Morb Mortal Wkly Rep. 2015;64(1):1-5.

56. Boon CS, Xu Z, Yue X, McClements DJ, Weiss J, Decker EA. Factors affecting lycopene oxidation in oil-in-water emulsions. J Agric Food Chem. 2008;56(4):1408-1414.

57. Waraho T, Cardenia V, Rodriguez-Estrada MT, McClements DJ, Decker EA. Prooxidant mechanisms of free fatty acids in stripped soybean oil-in-water emulsions. J Agric Food Chem. 2009;57(15):7112-7117.

58. Choi SW, Benzie IF, Collins AR, Hannigan BM, Strain JJ. Vitamins C and E: acute interactive effects on biomarkers of antioxidant defence and oxidative stress. Mutat Res. 2004;551(1-2):109-117.

59. Havel RJ, Eder HA, Bragdon JH. The distribution and chemical composition of ultracentrifugally separated lipoproteins in human serum. J Clin Invest. 1955;34(9):1345-1353.

60. Kono N, et al. Impaired $\alpha$-TTP-PIPs interaction underlies familial vitamin E deficiency. Science. 2013;340(6136):1106-1110.

61. Bartels M, Biesalski HK, Engelhart K, Sendlhofer G, Rehak P, Nagel E. Pilot study on the effect of parenteral vitamin E on ischemia and reperfusion induced liver injury: a double blind, randomized, placebo-controlled trial. Clin Nutr. 2004;23(6):1360-1370.

62. Leonard SW, Bruno RS, Ramakrishnan R, Bray T, Traber MG. Cigarette smoking increases human vitamin E requirements as estimated by plasma deuterium-labeled CEHC. Ann N Y Acad Sci. 2004;1031:357-360.

63. Kenward MG, Roger JH. An improved approximation to the precision of fixed effects from restricted maximum likelihood. Comput Stat Data Anal. 2009;53(7):2583-2595. 